\title{
Modelling the Impact of Screening on the Transmission Dynamics of Human Papillomavirus with Optimal Control
}

\author{
ESHETU DADI GURMU*, BOKA KUMSA BOLA, PURNACHANDRA RAO KOYA \\ Department of Mathematics \\ Wollega University \\ Nekemte \\ ETHIOPIA
}

\begin{abstract}
In this study, a nonlinear deterministic mathematical model of Human Papillomavirus was formulated. The model is studied qualitatively using the stability theory of differential equations. The model is analyzed qualitatively for validating the existence and stability of disease free and endemic equilibrium points using a basic reproduction number that governs the disease transmission. It's observed that the model exhibits a backward bifurcation and the sensitivity analysis is performed. The optimal control problem is designed by applying Pontryagin maximum principle with three control strategies viz. prevention strategy, treatment strategy, and screening strategy. Numerical results of the optimal control model reveal that a combination of prevention, screening, and treatment is the most effective strategy to wipe out the disease in the community.
\end{abstract}

\section{Key-Words: Model, stability, Simulation, Equilibrium, Control}

Received: July 7, 2021. Revised: December 3, 2021. Accepted: December 15, 2021. Published: December 28, 2021.

\section{Introduction}

Human Papillomavirus (HPV) is the name of a group of viruses that includes more than 100 different types and also further than 40 of these viruses are the most common and sexually transmitted in the world [1]. Most of the HPV infections are asymptomatic and can feed out without treatment over the course of a few times. For case, about 70 of HPV infections fed down within a time and 90 within two times. Still, in some people, the infection can persist for numerous times and can beget knobs or low risk genotype of HPV, while other types lead to different kinds of cancers or high risk genotype of HPV including cervical cancer [23].

Statistics show that18.1 million new cases, 9.6 million cancer related deaths, and 43.8 million people living with cancer in 2018 . The number of new cases is anticipated to rise from 18 million to 22 million by 2030 and the number of global cancer deaths is projected to increase by 45 in the period from 2007 to 2030 [4]. Nearly 80 of cervical cancer cases and deaths do in poor countries.

In Sub Saharan Africa, cervical cancer accounts for 22.5 of all cancer cases in women and the majority of women who develop cervical cancer live in pastoral areas [5]. Eastern Africa is one of the most heavily affected areas with an prevalence of more than 30 cases per women per year [6]. In Ethiopia, cancer accounts for about5.8 of total national mortality. Although population based data doesn't live in the country except for Addis Ababa, it's estimated that the periodic prevalence of cancer is around cases and the periodic mortality over. The most current cancers in Ethiopia among the entire adult population are breast cancer $[3,2]$ cancer of the cervix [13, 4], and colorectal cancer [5, 7]. About two thirds of annual cancer deaths do among women [7].

Numerous mathematical models have been developed to dissect the dynamics of transmission of HPV infection and its associated health problems, and as well as study the impact of some control strategies against the contagion. It's an essential and effective way to completely understand real world problems by establishing mathematical models and analyzing their dynamical behaviours. Old and recent studies similar as $[8,9]$ amongst others have shown that mathematical modelling is a extensively used tool for resolving questions on public health. Several SIR models [1012] have been developed to assess the implicit impact of vaccination against Human Papillomavirus. Also, [13, 14] formulated an SIS model for Human Papillomavirus transmission with vaccination as a control strategy, and [15] developed a dynamic model for the heterosexual transmission of Human Papillomavirus types 16 and 18 , which are covered by available 
vaccines. Also, some other recent studies by Akram et al [16] develop the mathematical model that describes the intercourse between uninfected tumour cells and infected tumour cells and modified with count treatment of cells by chemotherapy and recovery class.

The aim of this work is to study the effect of incorporating optimal control strategies to the sensitivity analysis and modelling the impact of screening on the transmission dynamics of Human Papillomavirus [18]. But the findings of this paper differ from the work presented in [18] because the model incorporates optimal control strategies.

\section{Model Description and Formulation}

The model divides the total population into five subclasses according to their disease status as: (i)

Susceptible subclass denoted by $S(t)$ consists of individuals which are capable of becoming infected

(ii) Unaware infected subclass denoted by $I_{u}(t)$ consists of individuals which are unaware infected with virus and are also infectious (iii) Screened infected subclass denoted by $I_{s}(t)$ consists of individuals which are screened infected with virus and provide treatment for those who are found to have HPV infection (iv) Recovered subclass denoted by $R(t)$ consists of recovered individuals and (v) Cervical Cancer subclass denoted by $C(t)$ consists of cervical cancer individuals.

Susceptible individuals are recruited into the population at a constant rate $\Pi$. Susceptible cells may acquire HPV infection at rate $\lambda$ when they come into effective contact with infectious individuals at the rate $\beta$ that may lead to infection. The force of infection in the model is given as $\lambda=\frac{\beta\left[L_{u}(t)+I_{S}(t)\right]}{N}$. The unaware infected cells are screened and join the screened infected class at a rate $\alpha$. Some of the unaware infected cells progress to cervical cancer at a rate $E$ and others recover naturally through body immune system at a rate $\delta$.

The screened infected cells are treated at a rate $\omega$ and move to recovery class or may progress to develop cervical cancer as a result of failure of the treatment used at a rate $\phi$ thus moving to cervical cancer class. Recovered cells revert to the susceptible class after losing their immunity at a rate $\varphi$. All infectious individuals die of infection at a rate $\xi$. All types of cells suffer natural mortality at a rate $\mu$. All parameters in the model are positive.

Upon including the basic assumptions together with the description of both model variables and parameters the schematic diagram of the modified model can be given as in Figure 1.

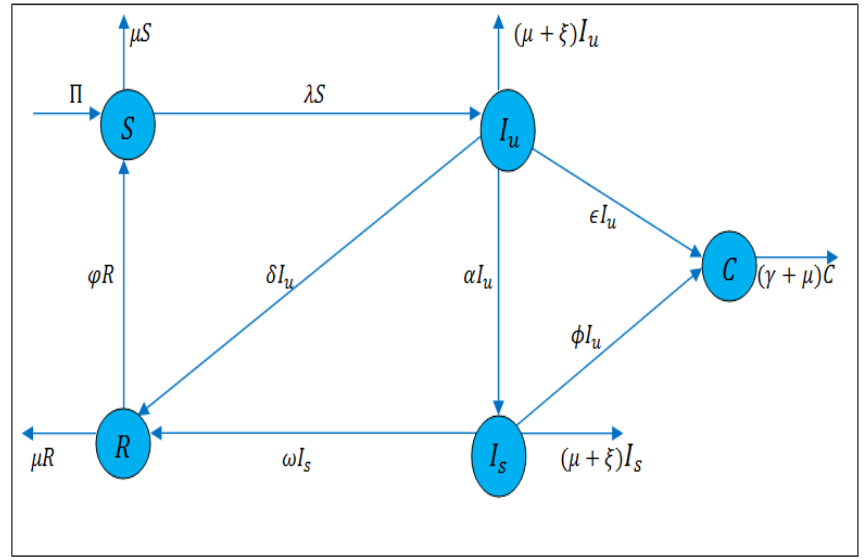

Fig. 1: Schematic Diagram of the Model HPV model

Based on the model assumptions, the notations of variables and parameters and the schematic diagram, the model equations are formulated and given as follows:

$$
\begin{aligned}
& \frac{d S}{d t}=\Pi+\varphi R-(\lambda+\mu) S \\
& \frac{d I_{u}}{d t}=\lambda S-(\epsilon+\alpha+\delta+\mu+\xi) I_{u} \\
& \frac{d I_{S}}{d t}=\alpha I_{u}-(\omega+\phi+\mu+\xi) I_{s}
\end{aligned}
$$




$$
\begin{gathered}
\frac{d C}{d t}=\epsilon I_{u}+\phi I_{s}-(\mu+\xi) C \\
\frac{d R}{d t}=\delta I_{u}+\omega I_{s}-(\varphi+\mu) R
\end{gathered}
$$

The non-negative initial conditions of the system of model equations (1) are denoted by

$$
\begin{aligned}
& S(0)=S_{0}, I_{u}(0)=I_{u 0}, I_{s}(0)=I_{s 0}, C(0)=C_{0}, \\
& R(0)=R_{0}
\end{aligned}
$$

\section{Mathematical Analysis of the Model}

\subsection{Invariant Region}

In this section, we obtain a region in which the solutions of model equation (1) are uniformly

bounded in the proper subsets of $\Omega \subset \mathbb{R}^{5}$. To obtain this, first we considered the total population $(N)$, where $N=S+I_{u}+I_{s}+C+R$. Then, after differentiating $(N)$ both sides with respect to $t$ and substituting the expression for $\frac{d S}{d t}, \frac{d I_{u}}{d t}, \frac{d I_{S}}{d t}, \frac{d C}{d t}$ and $\frac{d R}{d t}$ from equation (1) we obtained;

$$
\frac{d N}{d t}=\Pi-\mu N-\xi\left(I_{u}+I_{s}+C\right)
$$

In the absence of mortality due to disease $(\xi=0)$, then equation (2) become

$$
\frac{d N}{d t} \leq \Pi-\mu N
$$

After solving equation (3) and equating it as time tends to infinity, we obtain $0 \leq N(t) \leq\left(\frac{\Pi}{\mu}\right)$. Hence, the feasible solution set of model equation (1) remains in the region:

$$
\Omega=\left\{\left(\begin{array}{lllll}
(S, & I_{w}, & I_{s}, & C & R
\end{array}\right) \in \Re^{5}: N \leq \frac{m}{\mu}\right\}
$$

\subsection{Existence of the Solution}

Lemma 1: (Existence) Solutions of the model equations (1) together with the initial conditions

$S(0)>0, I_{u}(0)>0, I_{s}(0)>0, C(0)>$

$0, R(0)>0$

exist in $\mathbb{R}_{+}^{5}$ i.e., the model variables $S(t), I_{u}(t), I_{s}(t), C(t)$ and $R(t)$ exist for all $t$ and will remain in $\mathbb{R}_{+}^{5}$.

Proof: The right hand sides of the system of equations (1) can be expressed as follows:

$f_{1}\left(S, \quad I_{w}, \quad I_{\Xi}, \quad C, \quad R\right)=\Pi+\varphi R-(\lambda+\mu) S$

$f_{2}\left(S, \quad I_{w}, \quad I_{s}, \quad C, R\right)=\lambda S-(\epsilon+\alpha+\delta+$ $\mu+\xi) I_{u}$

$f_{3}\left(S, \quad I_{w}, \quad I_{g}, \quad C, R\right)=\alpha I_{u}-(\omega+\phi+\mu+$ ६) $I_{s}$

$f_{4}\left(S, \quad I_{w}, \quad I_{s}, \quad C, \quad R\right)=\epsilon I_{u}+\phi I_{s}-(\mu+\xi) C$ $f_{5}\left(S, \quad I_{w}, \quad I_{s}, \quad C, \quad R\right)=\delta I_{u}+\omega I_{s}-(\varphi+\mu) R$ According to Derrick and Groosman theorem, let $\quad \Omega \quad$ denote the region

$$
\left.\begin{array}{l}
\Omega=\left\{\left(\begin{array}{lllll}
S, & I_{w}, & I_{\Xi}, & C, & R
\end{array}\right) \in \mathbb{R}^{5}: N \leq\right. \\
\frac{\Pi}{\mu}
\end{array}\right\}
$$

. Then equations (1) have a unique solution if $\left(\partial f_{i}\right) /\left(\partial x_{j}\right), i, j=1,2,3,4,5$ are

continuous and bounded in $\Omega$.

Here, $x_{1}=S, x_{2}=I_{w}, x_{3}=I_{s}, x_{4}=C$

and $x_{5}=R$. The continuity and the boundedness are verified as follows: 
$\left|\left(\partial f_{1}\right) /(\partial S)\right|=|-(\lambda+\mu)|<\infty$,

$\left\|\left(\partial f_{1}\right) /\left(\partial I_{u}\right) \mid=\right\|(-\beta S / N) \|<\infty$,

$\|\left(\partial f_{1}\right) /\left(\partial I_{s}\right)|=|(-\beta S / N) \mid<\infty$,

$\|\left(\partial f_{1}\right) /(\partial C) \mid=0<\infty$,

$\left|\left(\partial f_{1}\right) /(\partial R)\right|=|\varphi|<\infty$.

$\|\left(\partial f_{2}\right) /(\partial S)|=| \lambda \mid<\infty$,

$\left\|\left(\partial f_{2}\right) /\left(\partial I_{w}\right)\right\|=\|(\beta S / N-(\alpha+\delta+\epsilon+$ $\mu+\xi) \mid<\infty$

$\left|\left(\partial f_{2}\right) /\left(\partial I_{s}\right)\right|=|(\beta S / N)|<\infty$,

$\left\|\left(\partial f_{2}\right) /(\partial C)\right\|=0<\infty$,

$\|\left(\partial f_{2}\right) /(\partial R) \mid=0<\infty$.

$\left|\left(\partial f_{3}\right) /(\partial S)\right|=0<\infty$,

$\left|\left(\partial f_{3}\right) /\left(\partial I_{w}\right)\right|=|\alpha|<\infty$

$\left|\left(\partial f_{3}\right) /\left(\partial I_{s}\right)\right|=|-(\omega+\phi+\mu+\xi)|<\infty$

$\left|\left(\partial f_{3}\right) /(\partial C)\right|=0<\infty$

$\left|\left(\partial f_{3}\right) /(\partial R)\right|=0<\infty$.

$\left\|\left(\partial f_{4}\right) /(\partial S)\right\|=0<\infty$,

$\left|\left(\partial f_{4}\right) /\left(\partial I_{w}\right)\right|=|\epsilon|<\infty$,

$\|\left(\partial f_{4}\right) /\left(\partial I_{s}\right)|=| \phi \mid<\infty$,

$\|\left(\partial f_{4}\right) /(\partial C)|=|-(\mu+\xi) \mid<\infty$

$\|\left(\partial f_{4}\right) /(\partial R) \mid=0<\infty$.

$\|\left(\partial f_{5}\right) /(\partial S) \mid=0<\infty$,

$\|\left(\partial f_{5}\right) /\left(\partial I_{u}\right)|=| \delta \mid<\infty$

$\|\left(\partial f_{5}\right) /\left(\partial I_{s}\right)|=| \omega \mid<\infty$,

$\left\|\left(\partial f_{5}\right) /(\partial C)\right\|=0<\infty$, $\left|\left(\partial f_{5}\right) /(\partial R)\right|=|-(\varphi+\mu)|<\infty$.

Thus, all the partial derivatives $\left(\partial f_{i}\right) /\left(\partial x_{j}\right), i, j=1,2,3,4,5 \quad$ exist, continuous

and bounded in $\Omega$. Hence, by Derrick and Groosman theorem, a solution for the model (1) exists and is unique.

\subsection{Positivity of the Solution}

In this section, we show all the solution of the model equation (1) remain positive for future time if their respective initial values are positive.

\section{Lemma}

2:

Let

$\Omega=\left\{\left(S, I_{w}, \quad I_{s}, C, R\right) \in \mathbb{R}_{+}^{5} ; S(0)>\right.$

$\left.0, I_{u}(0)>0, I_{s}(0)>0, C(0)>0, R(0)>0\right\}$

; then the solutions of $\left\{\begin{array}{lllll}S, & I_{w} & I_{s} & C, & R\end{array}\right\}$ are positive for all $t \geq 0$.

Proof: Positivity is verified separately for each of the model $S(t), \quad I_{u}(t), \quad I_{s}(t), \quad C(t)$ and $R(t)$.

Positivity of $S(t)$ : From model equation (1) we have:

$$
\begin{aligned}
& \frac{d S}{d t}= \Pi+\psi R-(\lambda+\mu) S, \\
& \Leftrightarrow \frac{d S}{d t} \geq-(\lambda+\mu) S, \\
& \Rightarrow \frac{d P}{S} \geq-(\lambda+\mu) d t, \\
& \Rightarrow \int \frac{d S}{S} \geq-\int(\lambda+\mu) d t_{,} \\
& \Rightarrow \ln S \geq-(\lambda+\mu) t+c_{4}, \\
& \Rightarrow S(t) \geq S_{0} \mathrm{e}^{-(\lambda+\mu) t}, S_{0}=\mathrm{e}^{c_{4}} \text { and } \\
& \mathrm{e}^{-(\lambda+\mu) t} \geq 0, \text { for all } t \geq 0 .
\end{aligned}
$$

Hence, it can be concluded that $S(t) \geq 0$. Similarly, we obtained in [18] as 
$\Rightarrow I_{u}(t) \geq I_{u 0} \mathrm{e}^{-(\alpha+\delta+\varepsilon+\mu+\xi) t}, I_{u 0}=\mathrm{e}^{c_{s}}$

and $\mathrm{e}^{-(\alpha+\delta+E+\mu+\xi) t} \geq 0$, for all $t \geq 0$.

$\Rightarrow I_{s}(t) \geq I_{s 0} \mathrm{e}^{-(\omega+\phi+\mu+\xi) t}, I_{s 0}=\mathrm{e}^{\mathrm{C}_{6}}$ and

$\mathrm{e}^{-(\omega+\phi+\mu+\xi) t} \geq 0$, for all $t \geq 0$.

$\Rightarrow C(t) \geq C_{0} \mathrm{e}^{-(\omega+\phi+\mu+\xi) t}, C_{0}=\mathrm{e}^{c_{7}}$ and

$\mathrm{e}^{-(\mu+\xi) t} \geq 0$, for all $t \geq 0$.

$\Rightarrow R(t) \geq R_{0} \mathrm{e}^{-(\varphi+\mu) t}, R_{0}=\mathrm{e}^{c_{\mathbb{E}}}$ and $\mathrm{e}^{-(\varphi+\mu) t} \geq 0$,

for all $t \geq 0$.

Therefore, the model variables

$S(t), \quad I_{u}(t), \quad I_{s}(t), \quad C(t)$ and $R(t)$ are positive

quantities and will remain in $\mathbb{R}_{+}^{5}$ for all $t \geq 0$.

\subsection{The Disease Free Equilibrium (DFE)}

Disease free equilibrium points are steady state solutions where there is no disease in the population. Absence of disease implies that

$I_{u}(t)=I_{s}(t)=C(t)=R(t)=0$ and the equilibrium points require that the right hand sides of the model equations set equal to zero. These requirements reflect in reducing the model equations (1) as

$$
\begin{array}{r}
\frac{d S}{d t}=\Pi+\varphi R-(\lambda+\mu) S \\
\Rightarrow \Pi-(\lambda+\mu) S=0 \\
\text { giving } S^{0}=\frac{\Pi}{(\lambda+\mu)}=\frac{\Pi}{\mu} \\
\text { where } \lambda=\frac{\beta\left(I_{u}+I_{S}\right)}{N}=\frac{\beta(0+0)}{N}=0 .
\end{array}
$$

Thus, the disease-free equilibrium point of the model equation in (1) above is given by

$$
\begin{aligned}
E_{0} & =\left\{S^{0}, I_{u}{ }^{0}, I_{s}{ }^{0}, C^{0}, R^{0}\right\} \\
& =\left\{\left(\frac{\Pi}{\mu}\right), 0,0,0,0\right\} .
\end{aligned}
$$

\subsection{The Basic Reproduction Number $\left(\Re_{0}\right)$}

The basic reproduction number is denoted by $\Re_{0}$ and is defined as the expected number of people getting secondary infection among the whole susceptible population. It is computed using the next-generation

matrix defined as in [17]. In this method $\Re_{0}$ is defined as the largest eigenvalue of the next generation matrix. Using the notation as in [17] for

the model system (1) the associated matrices $F$ and

$V$ for the new infectious terms and the remaining transition terms are respectively given by:

$$
\begin{gathered}
F_{i}=\left[\begin{array}{c}
{\left[\beta\left(I_{u}+I_{s}\right) S\right] / N} \\
0 \\
0
\end{array}\right] \text { and } \\
V_{i}=\left[\begin{array}{c}
(\epsilon+\alpha+\delta+\mu) I_{u} \\
-\alpha I_{u}+(\omega+\phi+\mu) I_{s} \\
-\epsilon I_{u}-\phi I_{s}+(\gamma+\mu) C
\end{array}\right]
\end{gathered}
$$

The Jacobian matrices of $F_{i}$ and $V_{i}$ at the disease free equilibrium point $E_{0}$ take the form respectively as

$$
F=\left[\begin{array}{lll}
\beta & \beta & 0 \\
0 & 0 & 0 \\
0 & 0 & 0
\end{array}\right] \text { and } V=\left[\begin{array}{ccc}
a & 0 & 0 \\
-\alpha & b & 0 \\
-\epsilon & -\phi & c
\end{array}\right]
$$

It can be verified that the matrix $V$ is non-singular as its determinant $\operatorname{det}[V]=a b c$ is non-zero and after some algebraic computations its inverse matrix is constructed as

$$
V^{-1}=\left[\begin{array}{ccc}
(1 / a) & 0 & 0 \\
(\alpha / a b) & (1 / b) & 0 \\
{[(\alpha \phi+\epsilon b) / \mu a]} & (\phi / b c) & (1 / c)
\end{array}\right]
$$

The product of the matrices $F$ and $V^{-1}$ can be computed as:

$F V^{-1}=\left[\begin{array}{lll}\beta & \beta & 0 \\ 0 & 0 & 0 \\ 0 & 0 & 0\end{array}\right]\left[\begin{array}{ccc}(1 / a) & 0 & 0 \\ (\alpha / a b) & (1 / b) & 0 \\ {[(\alpha \phi+\epsilon b) / \mu a]} & (\phi / b c) & (1 / c)\end{array}\right]=\left[\begin{array}{ccc}{[(\beta / a)+(\beta \alpha / a b)]} & (\beta / b) & 0 \\ 0 & 0 & 0 \\ 0 & 0 & 0\end{array}\right]$ Now it is possible to calculate the eigenvalue to determine the basic reproduction number $\Re_{0}$ by 
taking the spectral radius of the matrix $F V^{-1}$. Thus, the eigenvalues are computed by evaluating $\operatorname{det}\left[F V^{-1}-\lambda I\right]=0$ or equivalently solving $\left|\begin{array}{ccc}{[(\beta / a)+(\beta \alpha / a b)]-\lambda} & (\beta / b) & 0 \\ 0 & -\lambda & 0 \\ 0 & 0 & -\lambda\end{array}\right|=0$

It reduces to the cubic equation for $\lambda_{\text {as }}$ $\lambda^{2}[[(\beta / a)+(\beta \alpha / a b)]-\lambda]=0$ giving the three eigenvalues

as $\lambda_{1}=[(\beta / a)+(\beta \alpha / a b)], \lambda_{2}=0, \lambda_{3}=0$.

However, the largest eigenvalue here is $\lambda_{1}=[(\beta / a)+(\beta \alpha / a b)]$ and is the spectral radius as the threshold value or the basic reproductive number. Thus, it can be concluded that the reproduction number of the model is

$$
\Re_{0}=[\beta(b+\alpha) / a b] .
$$

\subsection{Local Stability of Disease Free Equilibrium}

In absence of the infectious disease, the model populations have a unique disease free steady

state $E_{0}$. To find the local stability of $E_{0}$, the Jacobian of the model equations evaluated at DEF

$E_{0}$ is used. It is already shown that the DFE of model (1) is given by $E_{0}=\left\{\left(\frac{\Pi}{\mu}\right), 0,0,0,0\right\}$. Now, the stability analysis of DEF is conducted and the results are presented in the form of theorems and proofs as follows:

Theorem 1: The DFE $E_{0}$ of the system (1) is locally asymptotically stable if $\Re_{0}<1$ and unstable if $\Re_{0}>1$.

Proof: Jacobian matrix of $\left(f_{1}, f_{2}, f_{3}, f_{4}, f_{5}\right)$ with respect to $\left(S, I_{w}, I_{s}, \quad C, R\right)$ is given by

$$
l=\left[\begin{array}{ccccc}
-\left[\left[\beta\left(I_{u}+I_{g}\right) / N\right]+\mu\right] & -(\beta S / N) & -(\beta S / N) & 0 & \varphi \\
{\left[\beta\left(I_{u}+I_{g}\right) / N\right]} & (\beta S / N)-a & (\beta S / N) & 0 & 0 \\
0 & a & -b & 0 & 0 \\
0 & \epsilon & \phi & -c & 0 \\
0 & \delta & \omega & 0 & -d^{d}
\end{array}\right] \text { (5) }
$$

Therefore, the Jacobian matrix $I$ of model at the disease free equilibrium $E_{0}$ reduces to

$I\left(E_{0}\right)=\left[\begin{array}{ccccc}-\mu & -\beta & -\beta & 0 & \varphi \\ 0 & (\beta-a) & \beta & 0 & 0 \\ 0 & \alpha & -b & 0 & 0 \\ 0 & \epsilon & \phi & -c & 0 \\ 0 & \delta & \omega & 0 & -d\end{array}\right]$

Now, the eigenvalues of $I\left(E_{0}\right)$ are required to be found. The characteristic equation $\operatorname{det}\left[J\left(E_{0}\right)-\psi I\right]=0$ is expanded and simplified as follows:

$$
\left|\begin{array}{ccccc}
-\mu-\psi & -\beta & -\beta & 0 & \varphi \\
0 & (\beta-a)-\psi & \beta & 0 & 0 \\
0 & \alpha & -b-\psi & 0 & 0 \\
0 & \epsilon & \phi & -c-\psi & 0 \\
0 & \delta & \omega & 0 & -d-\psi
\end{array}\right|=0
$$

$$
\begin{aligned}
& (\mu+\psi)(d+\psi)(-c-\psi)\left[\psi^{2}+(a+b-\beta) \psi+a b\left(1-\Re_{0}\right)\right]=0 \\
& (\mu+\psi)=0, \quad(d+\psi)=0, \\
& (-c-\psi)=0,
\end{aligned}
$$

$\psi^{2}+(a+b-\beta) \psi+a b\left(1-\Re_{0}\right)=0$

Thus, the five eigenvalues of the matrix are determined as

$\psi_{1}=-\mu, \psi_{2}=-d, \psi_{3}=-c$

$\psi_{4}=\frac{-(a+b-\beta)+\sqrt{(a+b-\beta)^{2}-4 a b\left(1-R_{0}\right)}}{2}$

$\psi_{5}=\frac{-(a+b-\beta)-\sqrt{(a+b-\beta)^{2}-4 a b\left(1-R_{0}\right)}}{2}$

It can be observed that the first three eigenvalues $\psi_{1}, \psi_{2}$ and $\psi_{3}$ are absolutely negative quantities. However, the remaining two $\psi_{4}$ and $\psi_{5}$ are also negatives so long as the following restrictions on the parameters are valid: $a b\left(1-\Re_{0}\right)>0 \quad$ and 
$(a+b-\beta)^{2}>2 a b\left(1-\Re_{0}\right)$ respectively, when $\Re_{0}<1$.

Therefore, it is concluded that the DFE $E_{0}$ of the system of differential equations (1) is locally asymptotically stable if $\Re_{0}<1$ and unstable if $\Re_{0}>1$.

\subsection{Global Stability of Disease Free Equilibrium}

The global stability of disease free equilibrium was implemented by Castillo-Chavez and Song technique [19]. The model equation (1) can be rewritten as

$d X / d t=F(X, Z)$

$$
d Z / d t=G(X, Z), \quad G(X, 0)=0
$$

Where, $X$ stands for the uninfected population, that is $X=(S, R)$ and $Z$ also stands for the infected population, that is $Z=\left(\begin{array}{lll}I_{w}, & I_{s}, & C\end{array}\right)$. The disease free equilibrium point of the model is denoted by $U=\left(X^{8}, 0\right)$. The point $U=\left(X^{8}, 0\right)$ to be globally asymptotically stable equilibrium for the model provided that $\Re_{0}<1$ and the following conditions must be met:

$\left(H_{1}\right) \quad$ For $d X / d t=F(X, 0), \quad X^{*} \quad$ is globally asymptotically stable.

$$
\begin{aligned}
& \left(H_{2}\right) G(X, Z)=A Z-\tilde{G}(X, Z), \\
& \qquad \tilde{G}(X, Z) \geq 0 \text { for }(X, Z) \in \Omega .
\end{aligned}
$$

Where $A=D_{Z} G(U, 0)$ a Metzler matrix is i.e. the off diagonal elements of $A$ are non-negative and $G$ is the region where the model makes biologically sense.If the model (1) met the above two criteria then the following theorem holds.
Theorem 2: The point $U=\left(X^{*}, 0\right)$ is globally asymptotically stable equilibrium provided that $\Re_{0}<1$ and the condition $\left(H_{1}\right)$ and $\left(H_{2}\right)$ are satisfied.

Proof: From system (1) we can get $F(X, Z)$ and $G(X, Z)$;

$$
\begin{aligned}
& F(X, Z)=\left[\begin{array}{c}
\Pi+\varphi R-(\lambda+\mu) S \\
\delta I_{u}+\omega I_{s}-(\varphi+\mu) R
\end{array}\right] \text { and } \\
& G(X, Z)=\left[\begin{array}{c}
\lambda S-(\alpha+\delta+\epsilon+\mu+\xi) I_{u} \\
\alpha I_{u}-(\omega+\phi+\mu+\xi) I_{s} \\
\epsilon I_{u}+\phi I_{s}-(\mu+\xi) C
\end{array}\right]
\end{aligned}
$$

Consider the reduced system

$$
\frac{d X}{d t} \|_{Z=0}=\left[\begin{array}{c}
\Pi-\mu S \\
0
\end{array}\right]
$$

From equation (6) above it is obvious that $X^{*}=\left\{\frac{\Pi}{\mu}, 0\right\}$ is the global asymptotic point. This can be verified from the solution, namely, $S=\frac{\Pi}{\mu}+\left[S(0)-\frac{\Pi}{\mu}\right] e^{-\mu t}$. As $t \rightarrow \infty$ the solution $S \rightarrow \frac{\Pi}{\mu}$ implying that the global convergence of (6) in $\Omega$. From the equation for infected compartments in the model we have:

$$
A=\left[\begin{array}{ccc}
\beta-(\alpha+\delta+\epsilon+\mu+\xi) & \beta & 0 \\
\alpha & -(\omega+\phi+\mu+\xi) & 0 \\
\epsilon & \phi & -(\mu+\xi)
\end{array}\right]
$$

Since $A$ is Metzler matrix, i.e. all off diagonal elements are nonnegative. Then, $G(X, Z)$ can be written as, $G(X, Z)=A Z-\tilde{G}(X, Z)$, where

$$
\tilde{G}(X, Z)=\left[\begin{array}{c}
\beta\left(I_{u}+I_{s}\right)\left(1-\frac{s}{N}\right) \\
0 \\
0
\end{array}\right]=\left[\begin{array}{l}
\tilde{G}_{1}(X, Z) \\
\tilde{G}_{2}(X, Z) \\
\tilde{G}_{3}(X, Z)
\end{array}\right]
$$


It follows that, in equation (7) $\tilde{G}_{1}(X, Y) \geq 0$ and $\tilde{G}_{2}(X, Y)=\tilde{G}_{3}(X, Y)=0$. Hence, $\tilde{G}(X, Y) \geq 0$. Therefore, condition $\left(H_{1}\right)$ and $\left(H_{2}\right)$ are satisfied and we conclude that $U$ is globally asymptotically stable for $\Re_{0}<1$.

\subsection{The Endemic Equilibrium}

Endemic equilibrium point $E_{1}$ is a steady state solution where the disease persists in the population. For the existence and uniqueness of endemic

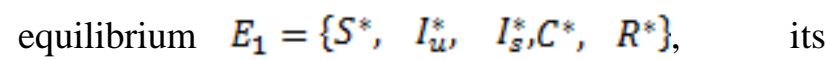
coordinates should satisfy the

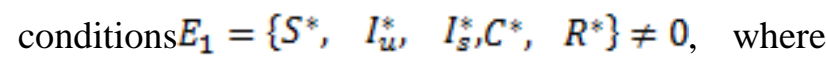
$S^{*}>0, I_{u}^{*}>0, I_{s}^{*}>0, C^{*}>0$ and $R^{*}>0$. The endemic equilibrium point is obtained by setting left hand sides of equations of the system (1) to zero. Then solved for state variables in terms of the force of infection, $\lambda^{*}$ and obtain the following;

$$
\begin{aligned}
S^{*} & =\frac{(a b d \Pi)}{\left[a b d\left(\lambda^{*}+\mu\right)-\lambda^{*} \varphi(b \delta+\omega \alpha)\right]} \\
I_{u}^{*} & =\frac{\left(b d \Pi \lambda^{*}\right)}{\left[a b d\left(\lambda^{*}+\mu\right)-\lambda^{*} \varphi(b \delta+\omega \alpha)\right]} \\
I_{s}^{*} & =\frac{\left(d \alpha \Pi \lambda^{*}\right)}{\left[a b d\left(\lambda^{*}+\mu\right)-\lambda^{*} \varphi(b \delta+\omega \alpha)\right]} \\
C^{*} & =\frac{\left[\alpha \Pi \lambda^{*}(\epsilon b+\phi \alpha)\right]}{\left[a b c d\left(\lambda^{*}+\mu\right)-c \lambda^{*} \varphi(b \delta+\omega \alpha)\right]} \\
R^{*} & =\frac{\left[\Pi \lambda^{*}(\delta b+\omega \alpha)\right]}{\left[a b d\left(\lambda^{*}+\mu\right)-\lambda^{*} \varphi(b \delta+\omega \alpha)\right]}
\end{aligned}
$$

Here $a=\epsilon+\alpha+\delta+\mu, b=\omega+\phi+\mu, c=\gamma+\mu$ and $d=\varphi+\mu$.

On substituting the expression for $I_{u}^{*}$ and $I_{s}^{*}$ into the force of infection, that is, $\lambda^{*}=\left[\beta\left(I_{u}^{*}+I_{u}^{*}\right)\right] / N$, characteristic polynomial of force of infection is obtained as

$p\left(\lambda^{*}\right)=D_{1} \lambda^{*}+D_{2}$

Here

$$
D_{1}=a b d-\varphi(b \delta+\omega \alpha)
$$

$\operatorname{and} D_{2}=\mu a b d\left(1-\Re_{0}\right)$.

Clearly, $\quad D_{1}>0 \quad$ and $D_{2} \geq 0$, when $[\varphi(b \delta+\omega \alpha)]<a b d \quad$ and

$\Re_{0}<1$,respectively and $\lambda^{*}=-D_{2} / D_{1} \leq 0$. From this, we see that, for, there is no endemic equilibrium for this model. Therefore, this condition shows that it is not possible for backward bifurcation in the model if $\Re_{0}<1$.

Lemma 3: A unique endemic equilibrium point $E^{*}$ exists and is positive if $\Re_{0}>1$.

\subsection{Global Stability Of Endemic Equilibrium}

Theorem 3: The endemic equilibrium point of the model equation (1) is globally asymptotically stable

whenever $\Re_{0}>1$.

Proof: To prove the global asymptotic stability of the endemic equilibrium we use the method of Lyapunov functions. Define

$$
\begin{aligned}
& L\left(S^{*}, I_{u}^{*}, I_{s}^{*}, C^{*}, R^{*}\right) \\
& \begin{array}{l}
=\left[S-S^{*}-S^{*} \ln \left(\frac{S^{*}}{S}\right)\right]+\left[I_{u}-I_{u^{*}}^{*}-I_{u}^{*} \ln \left(\frac{I_{u}^{*}}{I_{u}}\right)\right]+\left[I_{s}-I_{s^{*}}^{*}-I_{s}^{*} \ln \left(\frac{I_{s}^{*}}{I_{s}^{*}}\right)\right] \\
+\left[C-C^{*}-C^{*} \ln \left(\frac{C^{*}}{C}\right)\right]+\left[R-R^{*}-R^{*} \ln \left(\frac{R^{*}}{R}\right)\right]
\end{array}
\end{aligned}
$$

By direct calculating the derivative of $L$ along the solution (1) we have

$$
\begin{aligned}
\frac{d L}{d t}= & {\left[\frac{S-S^{*}}{S}\right] \frac{d S}{d t}+\left[\frac{I_{u}-I_{L^{*}}{ }^{*}}{I_{u}}\right] \frac{d I_{u}}{d t}+\left[\frac{I_{S}-I_{S}{ }^{*}}{I_{S}}\right] \frac{d I_{S}}{d t}+} \\
& {\left[\frac{C-C^{*}}{C}\right] \frac{d C}{d t}+\left[\frac{R-R^{*}}{R}\right] \frac{d R}{d t} }
\end{aligned}
$$

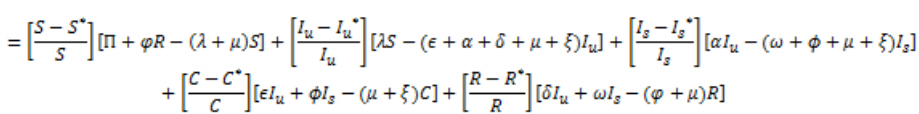




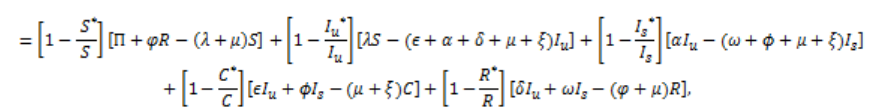

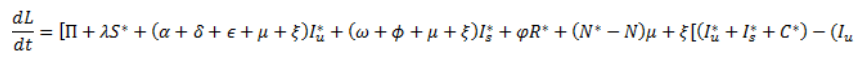
$\left.\left.+I_{u}+C\right)\right]-\left[(\Pi+\varphi R)\left(\frac{S^{*}}{S}\right)+\lambda\left(\frac{I_{u}^{*}}{I_{u}}\right)+\alpha I_{u}\left(\frac{I_{s}}{I_{s}}\right)+\left(\epsilon I_{u}+\phi I_{s}\right)\left(\frac{C^{*}}{C}\right)+\left(\delta I_{u}+\omega I_{s}\right)\left(\frac{R^{*}}{R}\right)\right]$

Thus collecting positive and negative terms together we obtain

$\frac{d L}{d t}=Q-K$. Here, $Q=\left[\Pi+\lambda S^{*}+(\alpha+\delta+\epsilon+\mu+\xi) I_{u}^{*}+(\omega+\phi+\mu+\xi) I_{s}^{*}+\varphi R^{*}+\left(N^{*}-N\right) \mu+\xi\left[\left(I_{u}^{*}+I_{s}^{*}+C^{*}\right)-\left(I_{u}\right.\right.\right.$
$\left.\left.\left.+I_{u}+C\right)\right]\right]$

And

$K=\left[(\Pi+\varphi R)\left(\frac{S^{*}}{S}\right)+\lambda S\left(\frac{I_{u}^{*}}{I_{u}}\right)+\alpha I_{u}\left(\frac{I_{s}^{*}}{I_{s}}\right)+\left(\epsilon I_{u}+\phi I_{s}\right)\left(\frac{C^{*}}{C}\right)+\left(\delta I_{u}+\omega I_{s}\right)\left(\frac{R^{*}}{R}\right)\right]$

Thus if $Q<K$, then $\frac{d L}{d t} \leq 0$. Noting that $\frac{d L}{d t}=0$ if and only

if

$S=S^{*}, I_{u}=I_{w}^{*}, I_{s}=I_{s}^{*}, C=C^{*}, R=R^{*}$.

Therefore, the largest compact invariant set in

$\left\{\left(S^{*}, I_{u}^{*}, I_{s}^{*}, C^{*}, R^{*}\right) \in \Omega: \frac{d L}{d t}=0\right\} \quad$ is the

singleton $E_{1}$ is the endemic equilibrium of the system (1). By LaSalle's invariant principle

(LaSalle's, 1976), it implies that $\mathrm{E}_{1}$ is globally

asymptotically stable in $\Omega$ if $Q<K$.

\section{Sensitivity Analysis of Model Parameters}

We carried out sensitivity analysis in order to determine the relative significance of model parameters on disease transmission. The analysis will enable us to find out parameters that have high impact on the basic reproduction number and which should be targeted by intervention strategies. We perform sensitivity analysis by calculating the sensitivity indices of the basic reproduction number

$\Re_{0}$ in order to determine whether HPV can be spread in the population or not. These indices tell us how crucial each parameter is on the transmission of the HPV. To investigate which parameters in the

model system (1) have high impact on the $\Re_{0}$, we apply the approach presented by [20].
The explicit expression of $\Re_{0}$ is given by $\Re_{0}=[\beta(b+\alpha) / a b]$. Since $\Re_{0}$ depends only on seven

parameters

$\beta=0.3, \delta=0.6, \epsilon=0.15, \omega=0.3, \mu=$ $0.02, \alpha=1.6, \phi=0.04$

we derive an analytical expression for its sensitivity to each parameter using the normalized forward sensitivity index as by Chitnis [20] as follows:

Table 1 . Sensitivity indices Table.

\begin{tabular}{|l|l|l|}
\hline $\begin{array}{l}\text { Parameter } \\
\text { Symbol }\end{array}$ & Sensitivity index & $\begin{array}{l}\text { Sensitivity } \\
\text { indices }\end{array}$ \\
\hline$\beta$ & $\Upsilon_{\beta}^{R_{0}}=\left[\partial R_{0} / \beta\right] \times\left[\beta / R_{0}\right]$ & +1 \\
\hline$\delta$ & $\Upsilon_{\delta}^{R_{0}}=\left[\partial R_{0} / \delta\right] \times\left[\delta / R_{0}\right]$ & 0.6979 \\
\hline$\epsilon$ & $\Upsilon_{\Xi}^{R_{0}}=\left[\partial R_{0} / \epsilon\right] \times\left[\epsilon / R_{0}\right]$ & 0.2020 \\
\hline$\omega$ & $\Upsilon_{\omega}^{R_{0}}=\left[\partial R_{0} / \omega\right] \times\left[\omega / R_{0}\right]$ & -0.3743 \\
\hline$\mu$ & $\Upsilon_{\mu}^{R_{0}}=\left[\partial R_{0} / \mu\right] \times\left[\mu / R_{0}\right]$ & -0.2972 \\
\hline$\alpha$ & $\Upsilon_{\alpha}^{R_{0}}=\left[\partial R_{0} / \alpha\right] \times\left[\alpha / R_{0}\right]$ & -0.0787 \\
\hline$\phi$ & $\Upsilon_{\phi}^{R_{0}}=\left[\partial R_{0} / \phi\right] \times\left[\phi / R_{0}\right]$ & -0.0684 \\
\hline
\end{tabular}

The sensitivity indices of the basic reproductive number with respect to main parameters are arranged orderly in Table 1 . Those parameters that

have positive indices i.e. $\beta, \delta$ and $\epsilon$ show that they have great impact on expanding the disease in the community if their values are increasing. Due to the reason that the basic reproduction number increases as their values increase, it means that the average number of secondary cases of infection increases in the community. Furthermore, those parameters in which their sensitivity indices are negative i.e.

$\omega, \mu, \alpha$ and $\phi$ have an influence of minimizing the burden of the disease in the community as their values increase while the others are left constant. And also as their values increase, the basic reproduction number decreases, which leads to minimizing the endemicity of the disease in the community. 


\section{Extension of Model into an Optimal \\ Control}

In this section, we apply optimal control strategies of the model equations (1). This helps to reduce the disease in the specified time. The optimal control model is an extension of HPV model (1) by including the following three controls defined as;

$u_{1}$ is the prevention efforts, that protect susceptible from contracting the disease.

$u_{2}$ is the screening for individuals which are unaware infected with virus.

$u_{3}$ is the treatment for individuals which are screen infected with virus.

After incorporating the controls into the model equations (1) we obtain the following equation;

$$
\begin{aligned}
& \frac{d S}{d t}=\Pi+\varphi R-\left(1-u_{1}\right) \frac{\beta\left[I_{u}(t)+I_{s}(t)\right]}{N} S-\mu S \\
& \frac{d I_{u}}{d t}=\left(1-u_{1}\right) \frac{\beta\left[I_{u}(t)+I_{s}(t)\right]}{N} S-\left(1-u_{2}\right)(\alpha+\varepsilon) I_{u}-\left(u_{2}+\delta\right) I_{u}-(\mu+\xi) I_{u} \\
& \frac{d I_{s}}{d t}=\left(1-u_{2}\right) \alpha I_{u}-\left(u_{a}+\omega\right) I_{s}-\left(1-u_{a}\right) \phi I_{s}- \\
& \quad(\mu+\xi) I_{s}
\end{aligned}
$$

$$
\begin{aligned}
& \frac{d C}{d t}=\left(1-u_{2}\right) e I_{u}+\left(1-u_{a}\right) \phi I_{s}-(\mu+\xi) C \\
& \frac{d R}{d t}=\left(u_{2}+\delta\right) I_{u}+\left(u_{a}+\omega\right) I_{s}-(\varphi+\mu) R
\end{aligned}
$$

Our main objective is to minimize the objective

function $J$ considering the cost of presentations and treatments. The goal of the adopted strategy is to reduce unawared infected individuals, screened infected individuals and individuals with cervical cancer. Mathematically, the optimal control problem consists of minimizing the objective functional $J$ on a fixed time interval $T$ takes the form;

$$
I\left(u_{1}, u_{2}, u_{3}\right)=\int_{0}^{T}\left(\begin{array}{c}
M_{1} I_{u}+M_{2} I_{s}+M_{3} C \\
+\frac{1}{2} \sum_{i=1}^{3} w_{i} u_{i}^{2}(t)
\end{array}\right) d t \rightarrow
$$

Subject to

$$
\begin{aligned}
& \int \frac{d S}{d t}=\Pi+\varphi R-\left(1-u_{2}\right) \frac{\beta\left[L_{u}(t)+I_{2}(t)\right]}{N} S-\mu S \\
& \frac{d I_{u}}{d t}=\left(1-u_{2}\right) \frac{\beta\left[L_{u}(t)+I_{s}(t)\right]}{N} S-\left(1-u_{2}\right)(\alpha+E) L_{u}-\left(u_{2}+\delta\right) L_{u}-(\mu+\xi) L_{u} \\
& \frac{d I_{x}}{d t}=\left(1-u_{2}\right) \alpha I_{\mu}-\left(u_{x}+\omega\right) I_{x}-\left(1-u_{z}\right) \phi I_{x}-(\mu+\xi) I_{x} \\
& \frac{d C}{d t}=\left(1-u_{2}\right) \in I_{\mu}+\left(1-u_{z}\right) \phi I_{z}-(\mu+\xi) C \\
& \frac{d R}{d t}=\left(u_{2}+\delta\right) I_{u}+\left(u_{x}+\omega\right) I_{x}-(\varphi+\mu) R
\end{aligned}
$$

With

initial

condition,

$$
S(0)=S_{0}>0, I_{u}(0)=I_{u 0}>0, I_{s}(0)=I_{s 0}>
$$$$
0, C(0)=C_{0}>0, R(0)=R_{0}>0
$$

and

$$
\begin{aligned}
& U=\left\{\left(u_{1}, u_{2}, u_{3}\right): 0 \leq u_{1(\min )} \leq u_{1} \leq u_{1(\max )} \leq\right. \\
& 1,0 \leq u_{2(\min )} \leq u_{2} \leq u_{2(\max )} \leq 1,0 \leq \\
& \left.u_{3(\min )} \leq u_{3} \leq u_{3(\max )} \leq 1, t \in[0, T], i=1,2,3\right\}
\end{aligned}
$$

Where $M_{1}, M_{2}, M_{3}, \frac{k_{1}}{2}, \frac{k_{2}}{2}$ and $\frac{k_{3}}{2}$ are positive weights that balance the size of the integrand terms to reduce the dominance of any of the term in the

integral. The constants $k_{1}, k_{2}$ and $k_{3}$ measures the cost or effort required for the implementation of each of the three control measures adopted while

$M_{1}, M_{2}$ and $M_{3}$ measures the relative importance of reducing the associated classes on the spread of the

disease. The parameter $T$ is the duration of time, in years of protection (presentations) and treatment progress.

We assumed that $0 \leq u_{1}<1$, since protecting the contact between the entire susceptible and infectious individuals are impossible in reality. In practice, protecting the entire society is impossible due to many factors such as financial and human resource

constraint. Similarly, $0 \leq u_{2}<1$, because efficient implementation of screening may not be in a proper

ways and $0 \leq u_{3}<1$, because due to the failure of treatment. Thus, the control takes values in the set $[0,1) \times[0,1) \times[0,1)=[0,1)$.

If $u_{i}=0, i=1,2,3$ then no control measure is taken and the model equation (8) is equivalent to 
(1). On the other hand, if $u_{i}=1, i=1,2,3$ implies our control is $100 \%$ success. In reality this case is not possible.

Hence, we seek the optimal controls $u_{1}^{*}, u_{2}^{*}, u_{3}^{*}$ such that

$I\left(u_{1}^{*}, \quad u_{2}^{*}, \quad u_{3}^{*}\right)=\min _{u_{1}, u_{2}, u_{1} \in U} J\left(u_{1}, u_{2}, u_{3}\right)$

Where $U$ is the set of admissible controls defined by

$\mho=$

$\left\{\begin{array}{c}\left(u_{1}, u_{2}, u_{3}\right): 0 \leq u_{1(\min )} \leq u_{1} \leq u_{1(\max )} \leq 1, \\ 0 \leq u_{2(\min )} \leq u_{2} \leq u_{2(\max )} \leq 1, \\ 0 \leq u_{3(\min )} \leq u_{3} \leq u_{3(\max )} \leq 1\end{array}\right\}$

\subsection{Existence of an Optimal Control}

Theorem 4: Consider the objective function $J(u)$ as

(9) with the set of admissible control $U$ subject to the system (8), then there exist an optimal control

$\left(u_{1}^{*}, u_{2}^{*}, u_{3}^{*}\right) \in \mho^{3} \quad$ such that

$I\left(u_{1}^{*}, u_{2}^{*}, u_{3}^{*}\right)=\min _{u_{1}, u_{2}, u_{3} \in U} J\left(u_{1}, u_{2}, u_{3}\right) \quad$ the following conditions are satisfied.

The set of controls and corresponding state variables is nonempty.

The admissible control set $U$ is convex and closed.

All the right hand sides of equations of system (1) are continuous, bounded above by a sum of bounded control and state, and can be written as a linear function of $u, v$ and $w$ with coefficients depending on time and state.

The integrand of the objective functional

$M_{1} I_{u}+M_{2} I_{s}+M_{3} C+\frac{w_{1} u_{1}^{2}}{2}+\frac{w_{2} u_{2}^{2}}{2}+\frac{w_{g} u_{a}^{2}}{2} \quad$ is convex.

The integrand of the objective functional is bounded below

$M_{1} I_{u}+M_{2} I_{s}+M_{3} C+\frac{w_{1} u_{1}^{2}}{2}+\frac{w_{2} u_{2}^{2}}{2}+\frac{w_{g} u_{g}^{n}}{2} \geq c_{1}+$ $c_{2}\left|u_{1}\right|^{\tau}+c_{3}\left|u_{2}\right|^{\tau}+c_{4}\left|u_{3}\right|^{\tau}$

where $c_{1}, c_{2}, c_{3}, c_{4}>0$ and $\tau>1$.
Proof: The non trivial requirement on the set of admissible controls and the set of end conditions are followed by Fleming and Rishel's [21] theorem.

Condition 1: Using theorem 3.2 of PicardLindelof, if $g(x, u, t)$ is bounded, continuous and Lipschitz in the state variables, then there exists a unique solution corresponding to every admissible

control $\mho$. Hence, for every $u_{i} \in \mho$ and the state variables, we have

$$
0<N \leq \frac{\Pi}{\mu}
$$

and non empty by model assumption. Furthermore, with the bounded done in (10) it implies that the state variable is continuous and bounded. Additionally, the partial derivative of the state variables $\frac{\partial g}{\partial x}$ exist and finite (i.e. are all continuous). Therefore, there exists a unique solution $\left(S, I_{w}, I_{g}, C, R\right)$ that satisfies the initial conditions. Hence, the set of controls and the corresponding state variables is nonempty and condition 1 is satisfied.

Condition 2: Assume that $u_{1}, u_{2}, u_{3} \in \mho$ such that $\left\|u_{1}\right\| \leq 1,\left\|u_{1}\right\| \leq 1$ and $\left\|u_{3}\right\| \leq 1$. Now, let us take any controls $u_{1}, u_{2} \in \mathcal{U}$ and $\lambda \in[0,1]$, then $0 \leq \lambda u_{1}+(1-\lambda) u_{2}$. Additionally, we observe that

$$
\begin{aligned}
& \left\|\lambda u_{1}\right\|=\lambda\left\|u_{1}\right\| \leq \lambda \text { and } \\
& \left\|(1-\lambda) u_{2}\right\|=(1-\lambda)\left\|u_{2}\right\| \leq(1-\lambda)
\end{aligned}
$$

Since, $\left\|u_{i}\right\| \leq 1$

Then for any $\lambda \in[0,1]$,

$$
\begin{aligned}
& \left\|\lambda u_{1}+(1-\lambda) u_{2}\right\| \\
& \leq\left\|\lambda u_{1}\right\|+\left\|(1-\lambda) u_{2}\right\| \\
& =\lambda\left\|u_{1}\right\|+(1-\lambda)\left\|u_{2}\right\|
\end{aligned}
$$


$<\lambda+(1-\lambda)=1$

Hence, $0 \leq \lambda u_{1}+(1-\lambda) u_{2} \leq 1_{\nu} \quad$ for $\quad$ all $u_{1}, u_{2} \in \mathcal{U}$ and $\lambda \in[0,1]$.

Therefore, the control space

$$
\begin{gathered}
\mho=c \\
\left\{\begin{array}{c}
\left(u_{1}, u_{2}, u_{3}\right):\left(u_{1}, u_{2}, u_{3}\right) \\
\text { is measurable, } \\
0 \leq u_{1(\min )} \leq u_{1} \leq u_{1(\max )} \leq 1, \\
0 \leq u_{2(\min )} \leq u_{2} \leq u_{2(\max )} \leq 1 \\
\\
0 \leq u_{3(\min )} \leq u_{3} \leq u_{3(\max )} \leq 1, t \in[0, T]
\end{array}\right\}
\end{gathered}
$$

is convex and closed by definition.

Condition 3: The right hand side of the model equation (1) satisfies condition 3 as the state solutions are a priori bounded.

Condition 4: The integrand in the objective functional, which is a cost functional,

$f(x, u, t)=M_{1} I_{u}+M_{2} I_{s}+M_{3} C+\frac{w_{1} u_{1}^{2}}{2}+\frac{w_{2} w_{2}^{2}}{2}+$ $\frac{w_{g} u_{g}^{2}}{2}$

is an affine function. Recall that any affine function is a convex and the sum of a convex function is a convex. Therefore, $f(x, u, t)$ is convex on $\mho$.

Condition 5: Assume that there exists constants $c_{1}, c_{2}, c_{3}, c_{4}>0$ and $\tau>1$ such that $M_{1} I_{u}+M_{2} I_{s}+M_{3} C+\frac{w_{1} w_{1}^{\pi}}{2}+\frac{w_{2} u_{2}^{\pi}}{2}+\frac{w_{g} u_{g}^{\pi}}{2}$ satisfies

$M_{1} I_{u}+M_{2} I_{s}+M_{3} C+\frac{w_{1} u_{1}^{2}}{2}+\frac{w_{2} u_{2}^{2}}{2}+\frac{w_{g} u_{u}^{2}}{2} \geq c_{1}+$ $c_{2}\left|u_{1}\right|^{\tau}+c_{3}\left|u_{2}\right|^{\tau}+c_{4}\left|u_{3}\right|^{\tau}$

Thus, the state variables are being bounded let

$$
c_{1}=\inf _{t \in[0, T]}\left(M_{1} I_{u}+M_{2} I_{s}+M_{3} C\right), c_{2}=
$$$$
\frac{w_{1}}{2}, c_{3}=\frac{w_{2}}{2}, c_{4}=\frac{w_{g}}{2}
$$

and $\tau=2$ then it follows that;
$M_{1} I_{u}+M_{2} I_{s}+M_{3} C+\frac{w_{1} u_{1}^{2}}{2}+\frac{w_{2} u_{2}^{2}}{2}+\frac{w_{3} u_{3}^{2}}{2} \geq c_{1}+c_{2}\left|u_{1}\right|^{\tau}+c_{3}\left|u_{2}\right|^{\tau}+c_{4}\left|u_{3}\right|^{\tau}$

Hence, conditions (1-5) are satisfied. Therefore, by Fleming and Rishel [21] we conclude that there

exists an optimal control $\left(x^{*}, u^{*}\right)$ that minimizes the cost functional over the set of admissible control $\widetilde{U}$.

\subsection{Characterization of an Optimal Control}

In order to derive the necessary conditions for the optimal control the Pontryagin's Maximum Principle [22] is used. According to the Pontryagin's

Maximum Principle, if $u^{*}(.) \in \mho$ with fixed final

time $T$, then there exists a non-trivial absolutely continuous mapping;

$\lambda:[0, T] \rightarrow \mathbb{R}^{5}, \lambda=\left(\lambda_{1}(t), \lambda_{2}(t), \lambda_{3}(t), \quad \lambda_{4}(t), \lambda_{5}(t)\right)$ are called the adjoint vector, such that

$I\left(u_{1}, u_{2}, u_{3}\right)=\int_{0}^{T}\left(M_{1} I_{u}+M_{2} I_{s}+M_{3} C+\frac{1}{2} \sum_{i=1}^{3} w_{i} u_{i}^{2}(t)\right) d t \rightarrow$ Min

Subject to

$\sum_{i=1}^{5} \lambda_{i}(t) g_{i}\left(S, I_{w}, I_{s}, C, R, u_{1}, u_{2}, u_{3}, t\right)$

Then, the Hamiltonian of the given system is defined as follows

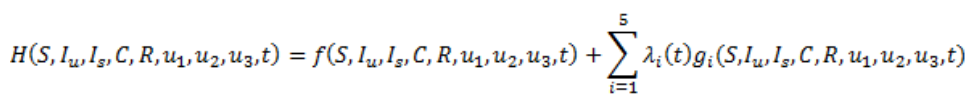

Where

$f\left(S, I_{w}, I_{s}, C, R, u_{1}, u_{2}, u_{3}, t\right)=\left(M_{1} I_{u}+M_{2} I_{s}+\right.$

$\left.M_{3} C+\frac{1}{2} \sum_{i=1}^{3} w_{i} u_{i}^{2}(t)\right)$

and $g_{i}(t)$ stands

for the right hand side of the constraints (8) for

$i=1,2,3,4,5$.

The optimality condition of the system,

$$
\frac{\partial H}{\partial u_{1}}=0, \frac{\partial H}{\partial u_{2}}=0, \frac{\partial H}{\partial u_{\mathrm{g}}}=0 .
$$

Hamiltonian system 
$\frac{d S}{d t}=\frac{\partial H}{\partial \lambda_{1}}, \frac{d I_{u}}{d t}=\frac{\partial H}{\partial \lambda_{2}}, \frac{d I_{S}}{d t}=\frac{\partial H}{\partial \lambda_{\mathrm{s}}}, \frac{d C}{d t}=\frac{\partial H}{\partial \lambda_{4}}, \frac{d R}{d t}=$

$\frac{\partial H}{\partial \lambda_{5}}$

Adjoint system

$\frac{d \lambda_{1}}{d t}=-\frac{\partial H}{\partial S}, \frac{d \lambda_{2}}{d t}=-\frac{\partial H}{\partial I_{u}}, \frac{d \lambda_{\mathrm{g}}}{d t}=-\frac{\partial H}{\partial I_{S}}, \frac{d \lambda_{4}}{d t}=$

$-\frac{\partial H}{\partial C}, \frac{d \lambda_{5}}{d t}=-\frac{\partial H}{\partial R}$

Minimality condition

$H\left(x^{*}(t), u^{*}(t), \lambda(t)\right)=\min H\left(x^{*}(t), u^{*}(t), \lambda(t)\right)$

holds for almost all $t \in[0, T]$.

Moreover, the transversality condition

$\lambda_{i}(T)=0, i=1,2,3,4,5$ also holds time.

Theorem 5: The optimal control problem (8) with fixed final time $T$ admits a unique optimal solution $\left(S^{*}, I_{w}^{*}, I_{s}^{*}, C^{*}, R^{*}\right)$ associated with an optimal control $u=\left(u_{1}, u_{2}, u_{3}\right)$ for all $t \in[0, T]$. Moreover, there exist adjoint functions $\lambda_{i}, i=1,2,3,4,5$ such that

$\frac{d \lambda_{1}}{d t}=\left(1-u_{1}\right) \frac{\beta\left[I_{u}(t)+I_{S}(t)\right]}{N}\left(\lambda_{1}-\lambda_{2}\right)+\mu \lambda_{1}$

$\frac{d \lambda_{2}}{d t}=-M_{1}+\left(1-u_{1}\right) \frac{\beta S}{N}\left(\lambda_{1}-\lambda_{2}\right)+\left[\left(1-u_{2}\right)(\alpha+\right.$ e) $\left.+\left(u_{2}+\delta\right)+(\mu+\xi)\right] \lambda_{2}-\left(1-u_{2}\right)\left[\alpha \lambda_{a}+\varepsilon \lambda_{4}\right]-$ $\lambda_{5}\left(u_{2}+\delta\right)$

$\frac{d \lambda_{3}}{d t}=-M_{2}+\left(1-u_{1}\right) \frac{\beta S}{N}\left(\lambda_{1}-\lambda_{2}\right)+\left[\left(1-u_{3}\right) \phi+\left(u_{3}+\omega\right)+(\mu+\xi)\right] \lambda_{3}-\left(1-u_{3}\right) \phi \lambda_{4}-\lambda_{5}\left(u_{3}+\omega\right)$

$\frac{d \lambda_{4}}{d t}=-M_{3}+\lambda_{4}(\mu+\xi)$

$\frac{d \lambda_{5}}{d t}=\lambda_{5}(\varphi+\mu)-\lambda_{1} \varphi$

With

transiversality

conditions $\lambda_{i}(T)=0, i=1,2,3,4,5$.
Similarly, we follow the approach of Pontryagin to get control. We solved the equation, $\frac{\partial H}{\partial u_{1}^{\circ}}=0$, at $u_{i}^{*}=0$ for $i=1,2,3$ and we obtain the control set $\left(u_{1}^{*}, u_{2}^{*}, u_{3}^{*}\right)$ characterized by;

$u_{1}^{*}=\max \left\{0, \min \left(1, \Phi_{1}\right)\right\}$

$u_{2}^{*}=\max \left\{0, \min \left(1, \Phi_{2}\right)\right\}$

$u_{3}^{*}=\max \left\{0, \min \left(1, \Phi_{3}\right)\right\}$

Where,

$\Phi_{1}=\frac{\lambda^{*} S\left(\lambda_{2}-\lambda_{1}\right)}{w_{1}}$

$\Phi_{2}=\frac{\left[\lambda_{2}+\epsilon\left(\lambda_{4}-\lambda_{2}\right)-\lambda_{5}\right] I_{u}}{w_{2}}$

$\Phi_{3}=\frac{\left[\lambda_{3}+\phi\left(\lambda_{4}-\lambda_{3}\right)-\lambda_{5}\right] I_{s}}{w_{3}}$

Proof: The Hamiltonian function associated with the system is defined as follows:

$H\left(S, I_{w}, I_{g}, C, R, u_{1}, u_{2}, u_{3}, t\right)=M_{1} I_{u}+M_{2} I_{s}+M_{3} C+\frac{1}{2} \sum_{i=1}^{3} w_{i} u_{i}^{2}(t)+\sum_{i=1}^{5} \lambda_{i}(t) g_{i}\left(S, I_{w}, I_{s}, C, R, u_{1}, u_{2}, u_{3}, t\right)$

$=M_{1} I_{u}+M_{2} I_{s}+M_{3} C+\frac{w_{1} u_{1}^{2}}{2}+\frac{w_{2} u_{2}^{2}}{2}+\frac{w_{3} u_{3}^{2}}{2}+\lambda_{1}\left[\Pi+\varphi R-\left(1-u_{1}\right) \lambda S-\mu S\right]$

$+\lambda_{2}\left[\left(1-u_{1}\right) \lambda S-\left(1-u_{2}\right)(\alpha+\epsilon) I_{u}-\left(u_{2}+\delta\right) I_{u}-(\mu+\xi) I_{u}\right]$

$+\lambda_{3}\left[\left(1-u_{2}\right) \alpha I_{u}-\left(u_{3}+\omega\right) I_{s}-\left(1-u_{3}\right) \phi I_{s}-(\mu+\xi) I_{s}\right]$

$+\lambda_{4}\left[\left(1-u_{2}\right) E I_{u}+\left(1-u_{3}\right) \phi I_{s}-(\mu+\xi) C\right]+\lambda_{5}\left[\left(u_{2}+\delta\right) I_{u}+\left(u_{3}+\omega\right) I_{s}-(\varphi+\mu) R\right]$

are the adjoint functions to be determined suitably. The form of the adjoint equations and transversality conditions are standard results from Pontryagin's Maximum Principle. We differentiate the

Hamiltonian with respect to states $S, I_{w}, I_{s,} C$ and

$R$ respectively and then the adjoint system can be obtained as follows:

$\frac{d \lambda_{1}}{d t}=-\frac{\partial H}{\partial S}=\left(1-u_{1}\right) \frac{\beta\left[I_{u}(t)+I_{g}(t)\right]}{N}\left(\lambda_{1}-\lambda_{2}\right)+\mu \lambda_{1}$ $\begin{aligned} \frac{d \lambda_{2}}{d t}=-\frac{\partial H}{\partial l_{u}}= & -M_{1}+\left(1-u_{1}\right) \frac{\beta S}{N}\left(\lambda_{1}-\lambda_{2}\right)+\left[\left(1-u_{2}\right)(a+\epsilon)+\left(u_{2}+\delta\right)+(\mu+\xi)\right] \lambda_{2}-\left(1-u_{2}\right)\left[a \lambda_{3}+\epsilon \lambda_{4}\right] \\ & -\lambda_{5}\left(u_{2}+\delta\right)\end{aligned}$

$\frac{d \lambda_{3}}{d t}=-\frac{\partial H}{\partial I_{g}}=-M_{2}+\left(1-u_{1}\right) \frac{\beta S}{N}\left(\lambda_{1}-\lambda_{2}\right)+\left[\left(1-u_{3}\right) \phi+\left(u_{3}+\omega\right)+(\mu+\xi)\right] \lambda_{3}-\left(1-u_{3}\right) \phi \lambda_{4}-\lambda_{5}\left(u_{3}+\omega\right)$

$\frac{d \lambda_{4}}{d t}=-\frac{\partial H}{\partial C}==-M_{a}+\lambda_{4}(\mu+\xi)$ 
$\frac{d \lambda_{5}}{d t}=-\frac{\partial H}{\partial R}=\lambda_{5}(\varphi+\mu)-\lambda_{1} \varphi$

Hence, following Lenhart, S. [23] the transversality conditions becomes $\lambda_{i}(T)=0$, for $i=1,2,3,4,5$.

By the optimality condition, we have: $\frac{\partial H}{\partial u_{i}}=0$, at

$u_{i}=u_{i}^{*}=0$ for $i=1,2,3$ we get

$\frac{\partial H}{\partial u_{1}}=0$, then $u_{1}^{*}(t)=\frac{\lambda^{*} S\left(\lambda_{2}-\lambda_{1}\right)}{w_{1}}$

$\frac{\partial H}{\partial u_{2}}=0$, then $u_{2}^{*}(t)=\frac{\left[\lambda_{2}+E\left(\lambda_{4}-\lambda_{2}\right)-\lambda_{5}\right] I_{u}}{w_{2}}$

$\frac{\partial H}{\partial u_{\mathrm{g}}}=0$, then $u_{3}^{*}(t)=\frac{\left[\lambda_{\mathrm{g}}+\phi\left(\lambda_{4}-\lambda_{\mathrm{g}}\right)-\lambda_{\mathrm{s}}\right] I_{\mathrm{S}}}{w_{\mathrm{g}}}$

When we write by using standard control arguments involving the bounds on the controls, we conclude;

$u_{1}^{*}=\left\{\begin{array}{ll}\Phi_{1}, & \text { if } 0<\Phi_{1}<1 \\ 0, & \text { if } \Phi_{1} \leq 0 \\ 1, & \text { if } \Phi_{1} \geq 1\end{array}\right.$,

$u_{2}^{*}=\left\{\begin{array}{ll}\Phi_{2}, & \text { if } 0<\Phi_{2}<1 \\ 0, & \text { if } \Phi_{2} \leq 0 \\ 1, & \text { if } \Phi_{2} \geq 1\end{array}\right.$,

$u_{3}^{*}=\left\{\begin{array}{ll}\Phi_{3}, & \text { if } 0<\Phi_{3}<1 \\ 0, & \text { if } \Phi_{3} \leq 0 \\ 1, & \text { if } \Phi_{3} \geq 1\end{array}\right.$.

In compact notation;

$u_{1}^{*}=\max \left\{0, \min \left(1, \Phi_{1}\right)\right\}$

$u_{2}^{*}=\max \left\{0, \min \left(1, \Phi_{2}\right)\right\}$

$u_{3}^{*}=\max \left\{0, \min \left(1, \Phi_{3}\right)\right\}$

Where,

$$
\begin{aligned}
& \Phi_{1}=\frac{\lambda^{*} S\left(\lambda_{2}-\lambda_{12}\right)}{w_{1}}, \\
& \Phi_{2}=\frac{\left[\lambda_{2}+E\left(\lambda_{4}-\lambda_{2}\right)-\lambda_{5}\right] I_{U}}{w_{2}}, \\
& \Phi_{3}=\frac{\left[\lambda_{\mathrm{g}}+\phi\left(\lambda_{4}-\lambda_{\mathrm{g}}\right)-\lambda_{5}\right] I_{\mathrm{s}}}{w_{\mathrm{g}}} .
\end{aligned}
$$

The optimality system is formed from the optimal control system (the state system) and the adjoint variable system by incorporating the characterized control set and initial and transversal condition, we obtained;

$$
\begin{aligned}
& \frac{d S}{d t}=\Pi+\varphi R-\left(1-u_{1}\right) \frac{\beta\left[I_{u}(t)+I_{s}(t)\right]}{N} S-\mu S \\
& \frac{d I_{u}}{d t}=\left(1-u_{1}\right) \frac{\beta\left[I_{u}(t)+I_{s}(t)\right]}{N} S-\left(1-u_{2}\right)(\alpha+\epsilon) I_{u}-\left(u_{2}+\delta\right) I_{u}-(\mu+\xi) I_{u} \\
& \frac{d I_{s}}{d t}=\left(1-u_{2}\right) a I_{u}-\left(u_{3}+\omega\right) I_{s}-\left(1-u_{3}\right) \phi I_{s}-(\mu+\xi) I_{s} \frac{d C}{d t}=\left(1-u_{2}\right) \in I_{u}+\left(1-u_{3}\right) \phi I_{s}-(\mu+\xi) C \\
& \frac{d R}{d t}=\left(u_{2}+\delta\right) I_{u}+\left(u_{3}+\omega\right) I_{s}-(\varphi+\mu) R \\
& \frac{d \lambda_{1}}{d t}=-\frac{\partial H}{\partial S}=\left(1-u_{1}\right) \frac{\beta\left[I_{u}(t)+I_{s}(t)\right]}{N}\left(\lambda_{1}-\lambda_{2}\right)+\mu \lambda_{1} \\
& \begin{aligned}
\frac{d \lambda_{2}}{d t}=-\frac{\partial H}{\partial I_{u}}=-M_{1}+\left(1-u_{1}\right) \frac{\beta S}{N}\left(\lambda_{1}-\lambda_{2}\right)+\left[\left(1-u_{2}\right)(\alpha+\epsilon)+\left(u_{2}+\delta\right)+(\mu+\xi)\right] \lambda_{2} \\
-\left(1-u_{2}\right)\left[\alpha \lambda_{3}+\epsilon \lambda_{4}\right]-\lambda_{5}\left(u_{2}+\delta\right)
\end{aligned} \\
& \begin{aligned}
\frac{d \lambda_{3}}{d t}=-\frac{\partial H}{\partial I_{s}}= & -M_{2}+\left(1-u_{1}\right) \frac{\beta S}{N}\left(\lambda_{1}-\lambda_{2}\right)+\left[\left(1-u_{3}\right) \phi+\left(u_{3}+\omega\right)+(\mu+\xi)\right] \lambda_{3}-\left(1-u_{3}\right) \phi \lambda_{4} \\
& -\lambda_{5}\left(u_{3}+\omega\right)
\end{aligned}
\end{aligned}
$$$$
\frac{d \lambda_{4}}{d t}=-\frac{\partial H}{\partial C}==-M_{3}+\lambda_{4}(\mu+\xi)
$$$$
\frac{d \lambda_{5}}{d t}=-\frac{\partial H}{\partial R}=\lambda_{5}(\varphi+\mu)-\lambda_{1} \varphi
$$

With initial condition:

$S(0)=S_{0}, I_{u}(0)=I_{u 0}, I_{s}(0)=I_{s 0}, C(0)=C_{0}$, $R(0)=R_{0}$

and

$$
\begin{aligned}
& \lambda_{1}(T)=0, \lambda_{2}(T)=0, \lambda_{3}(T)=0, \lambda_{4}(T)=0, \\
& \lambda_{5}(T)=0
\end{aligned}
$$

\section{Numerical Simulation}

In this section, the result obtained by numerically solving the optimality system was presented. In our control problem, we have initial conditions for the state variables and terminal conditions for the adjoints. That is, the optimality system is a twopoint boundary value problem with separated

boundary conditions at times step $i=0$ and $i=T$. The numerical simulation was carried out using the software MATLAB 2015b. To conduct the study, a set of meaningful values are assigned to the model parameters. These values are either taken from literature or assumed. Using the parameter values given in Table 2 and the initial conditions 
$S(0)=150000, I_{u}(0)=50000, I_{s}(0)=$ $30000, C(0)=12500$

and $R(0)=26250$ the simulation study is conducted and the results are given below in Figures.

Table 2. Parameter values used in Numerical Simulations

\begin{tabular}{|c|c|c|}
\hline Parameter & Value & Reference \\
\hline$\Pi$ & 0.004 & Assumed \\
\hline$\mu$ & 0.02 & {$[17]$} \\
\hline$\beta$ & 0.3 & {$[18]$} \\
\hline$\varphi$ & 0.2 & {$[18]$} \\
\hline$\alpha$ & 1.6 & {$[18]$} \\
\hline$\xi$ & 0.0001 & assumed \\
\hline$\omega$ & 0.3 & {$[18]$} \\
\hline$\epsilon$ & 0.15 & {$[18]$} \\
\hline$\delta$ & 0.6 & {$[18]$} \\
\hline$\phi$ & 0.04 & {$[18]$} \\
\hline
\end{tabular}

\section{a) Control strategy with prevention only}

We simulated the optimality control system by incorporating prevention intervention only. Figures 2(a), 2(b) and 2(c) shows that the decrease of all infectious individuals in the specified time but they did not go to zero over the period of implementation of this intervention strategy. The reason is that due to lack of prevention susceptible individuals still get infected. Therefore, we conclude that applying optimized prevention only as control intervention decreases the burden of the disease but it is not eradicate HPV totally from the community.

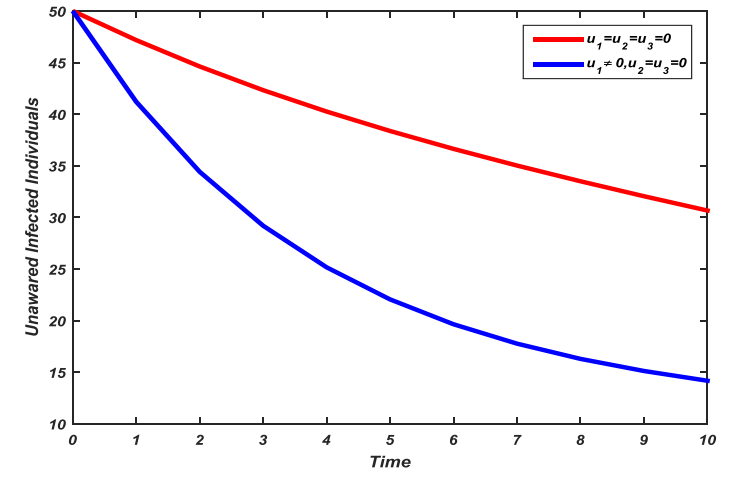

Fig. 2(a):, Simulations of unawared individuals with prevention only

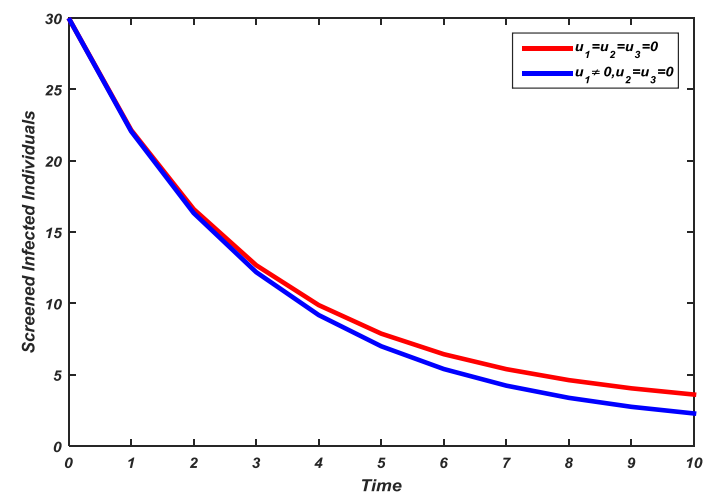

Fig. 2(b): Simulations of Screened individuals with prevention only

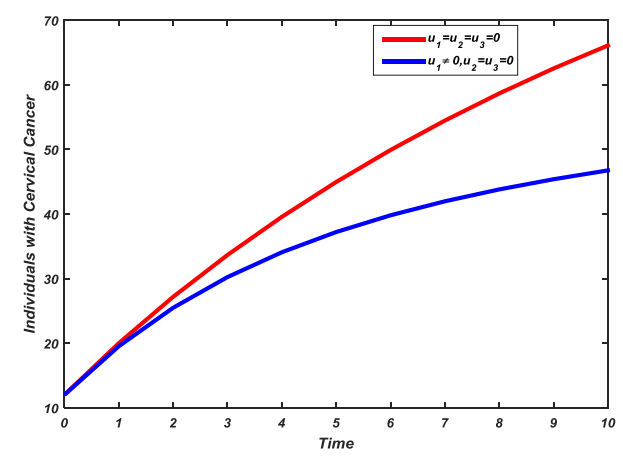

Fig. 2(c): Simulations of Cervical Cancer individuals with prevention only

\section{b) Control strategy with Screening only}

As we know screening helps unawared individuals to identify their status as they are leaving with the virus or not. Therefore, Figures 3(a), 3(b) and 3(c) shows that all infectious individuals go down by screening effort but their number cannot be zero. New infection always appears in the community because the diseases are not prevented and individuals who develop the symptom of the disease are not getting treatment. Therefore, control with screening only reduces the burden in some extent but it is not eradicate HPV totally from the community. 


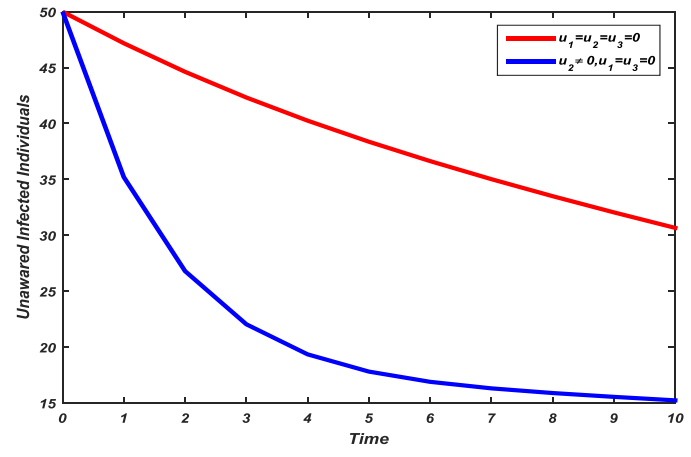

Fig. 3(a): Simulations of unawared individuals with screening only

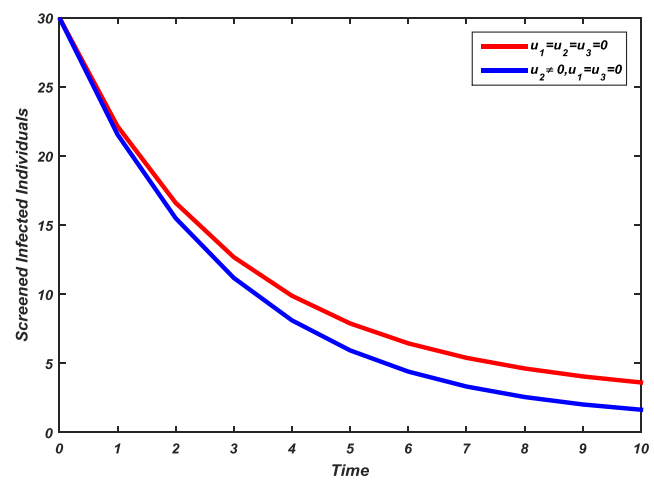

Fig. 3(b): Simulations of screened individuals with screening only

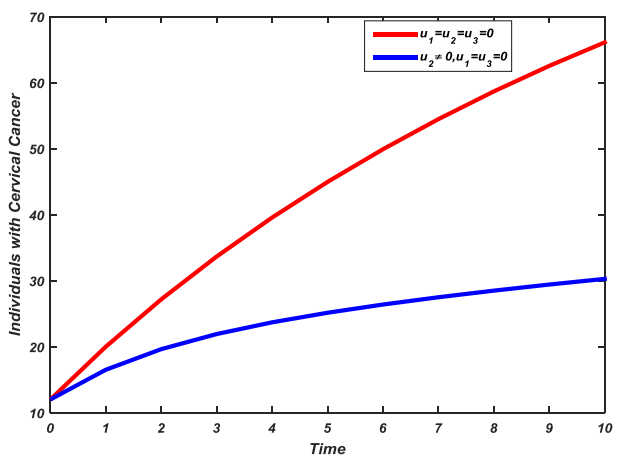

Fig. 3(c): Simulations of Cervical Cancer individuals with screening only

\section{c. Control strategy with treatment only}

We applied treatment only as intervention that is treating individuals who develop disease symptom. Figures 4(a), 4(b) and 4(c) clearly show that all infectious individuals have gone to zero at the end of the implementation period. Therefore, we conclude that this strategy is effective in eradicating the HPV from the community in a specified period of time.

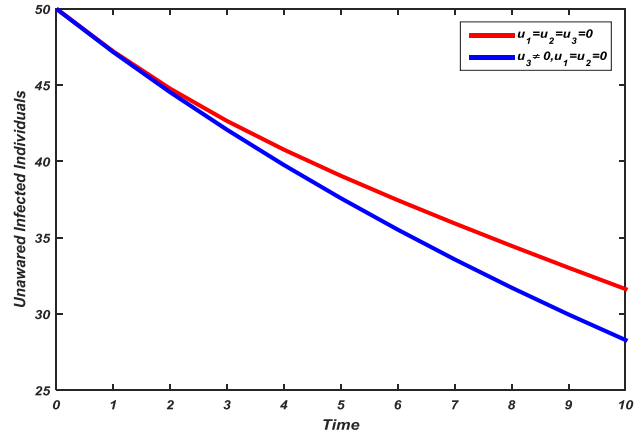

Fig. 4(a): Simulations of unawared individuals with treatment only

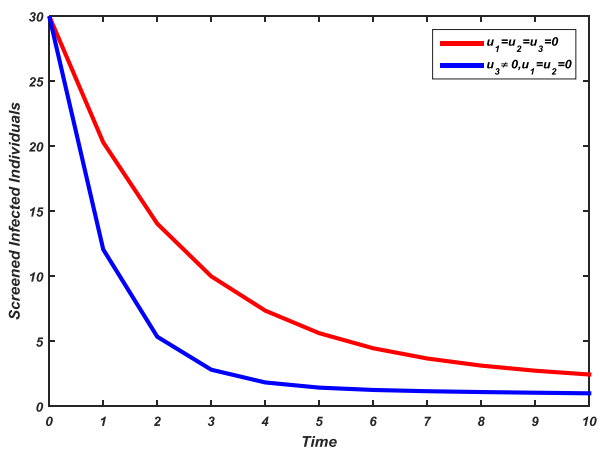

Fig. 4(b): Simulations of screened individuals with treatment only

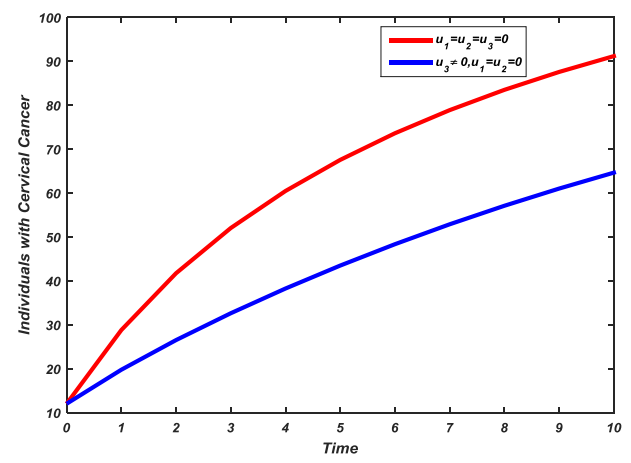

Fig. 4(c): Simulations of Cervical Cancer individuals with treatment only

\section{d. Control strategy with prevention and Screening only}

In this strategy, we applied prevention and screening as intervention to control HPV. Figures 5(a), 5(b) and 5(c) shows that infectious individuals did not goes to zero over the period of implementation of this intervention strategy. The reason is that due to lack of prevention susceptible individuals still get infected and due to lack of screening unawared individuals. Therefore, control with prevention and screening reduces the burden to some extent but it is not eradicate HPV totally from the community. 


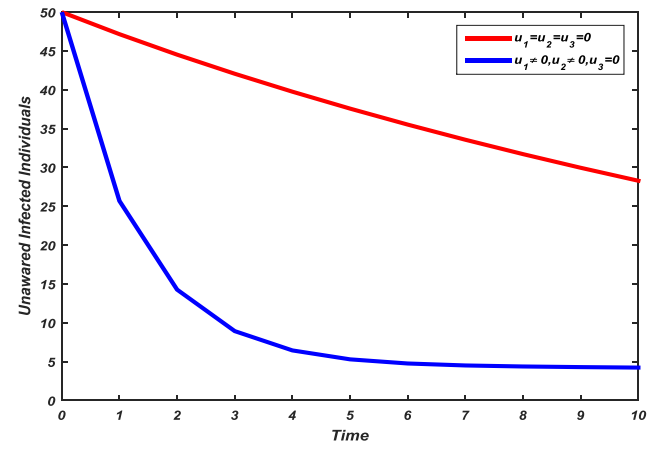

Fig. 5(a): Simulations of unawared individuals with prevention and screening only

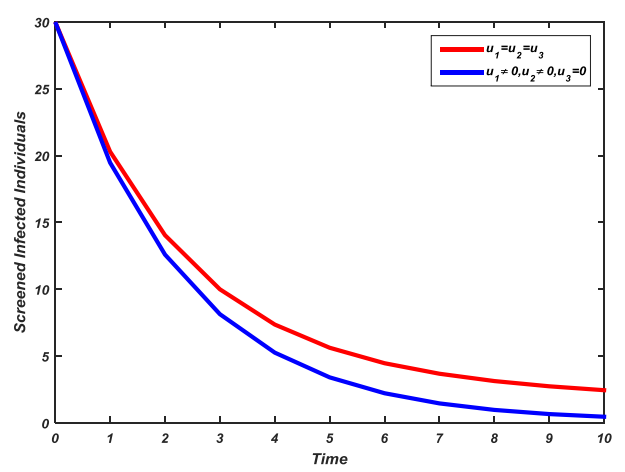

Fig. 5(b): Simulations of unawared individuals with prevention and screening only

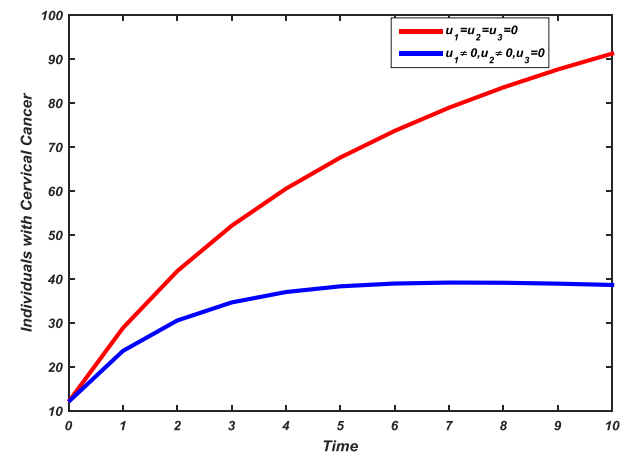

Fig. 5(c): Simulations of Cervical Cancer individuals with prevention and screening only

\section{e) Control strategy with prevention and treatment only}

We simulate the model using a combination of prevention and treatment as intervention strategy for control of HPV in the community. Figures 6(a), 6(b) and 6(c) shows that infectious individuals did not go to zero over the period of implementation of this intervention strategy. The reason is that due to lack of prevention susceptible individuals still get infected and due to lack of treatment individuals develop disease symptom. Therefore, this strategy is not $100 \%$ effective in eradicating the HPV in the specified period of time.

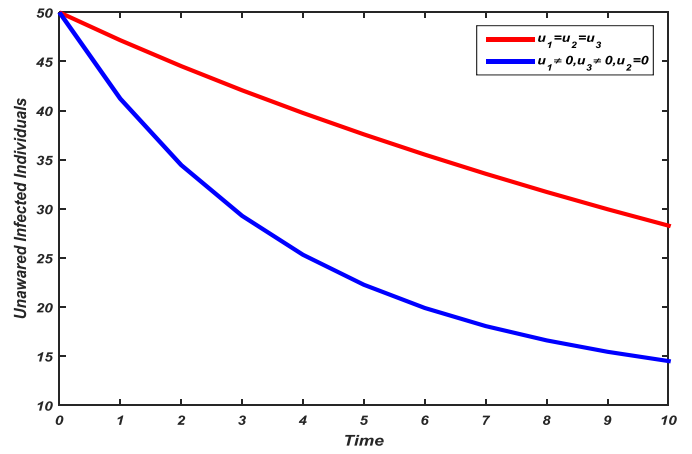

Fig. 6(a): Simulations of unawared individuals with prevention and treatment only

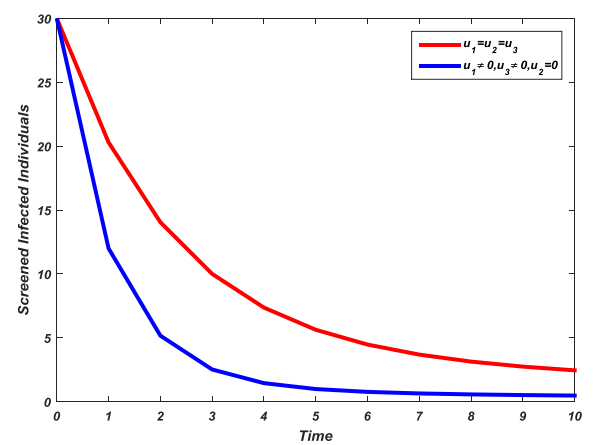

Fig. 6(b): Simulations of screened individuals with prevention and treatment only

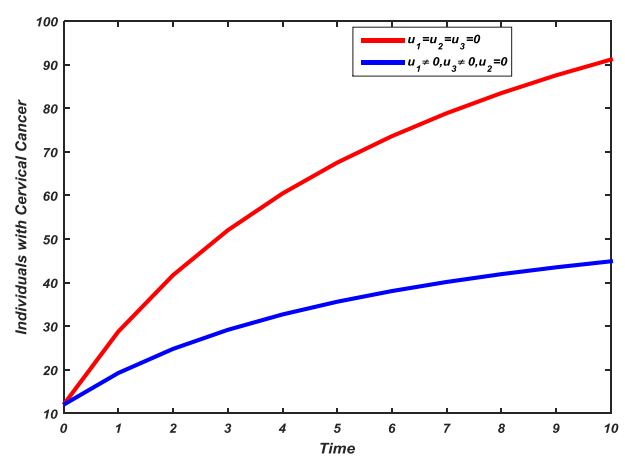

Fig. 6(c): Simulations of Cervical Cancer individuals with prevention and treatment only

\section{f) Control strategy with screening and treatment only}

We simulate the model using a combination of screening and treatment as intervention strategy for control of HPV in the community. Figures 7(a), 7(b) and $7(\mathrm{c})$ clearly show that infectious individuals have gone to zero at the end of the implementation period. Therefore, we conclude that this strategy is effective in eradicating HPV from the community in a specified period of time. 


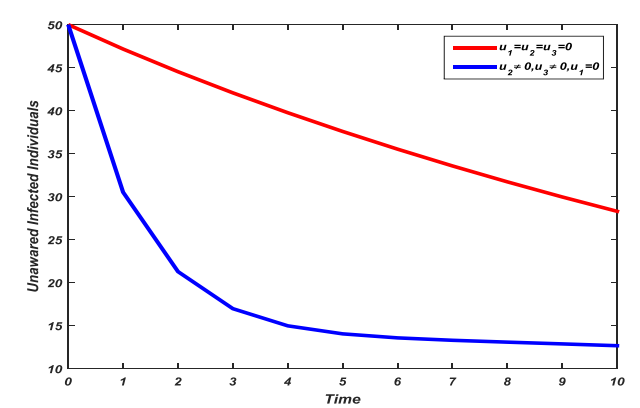

Fig. 7(a): Simulations of unawared individuals with screening and treatment only

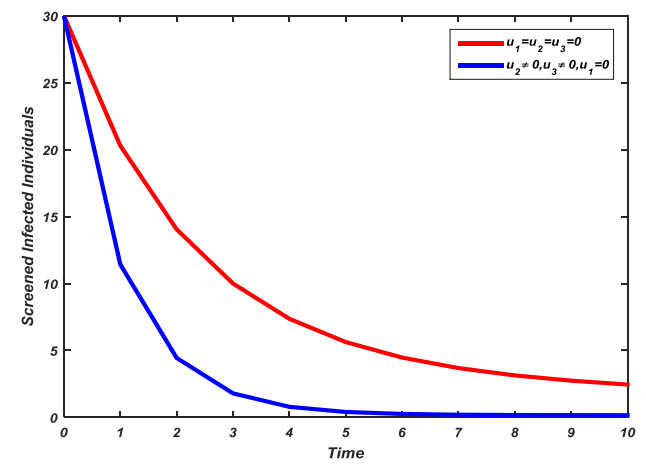

Fig. 7(b): Simulations of screened individuals with screening and treatment only

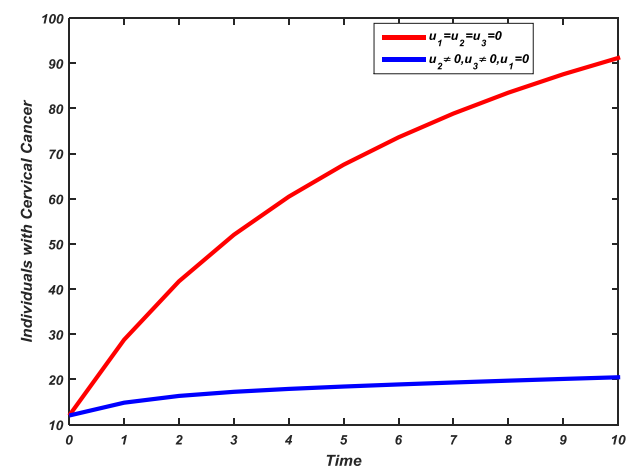

Fig. 7(c): Simulations of Cervical Cancer individuals with screening and treatment only

\section{g. Control strategy with prevention, screening and treatment}

In this strategy, we implemented all the three controls (prevention, screening and treatment) as intervention to eradicate HPV from the community. Figures 8(a), 8(b) and 8(c) shows that an infectious individual goes to zero at the end of the implementation period. Therefore, applying this strategy is effective in eradicating HPV form the community in a specified period of time.

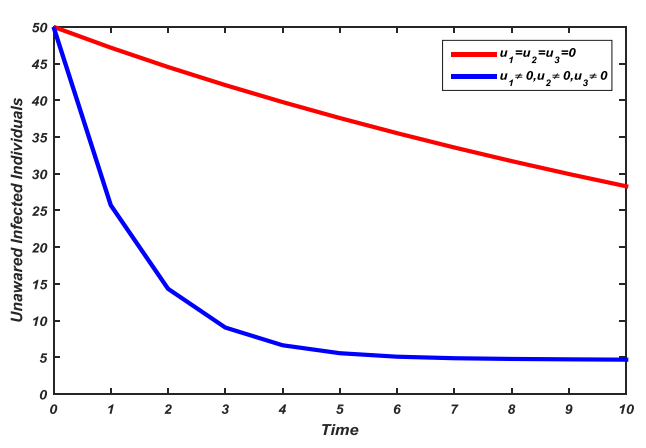

Fig. 8(a): Simulations of unawared individuals with all controls

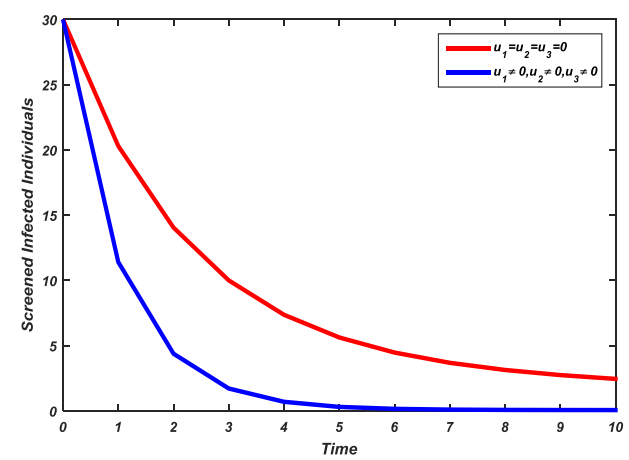

Fig. 8(b): Simulations of screened individuals with all controls

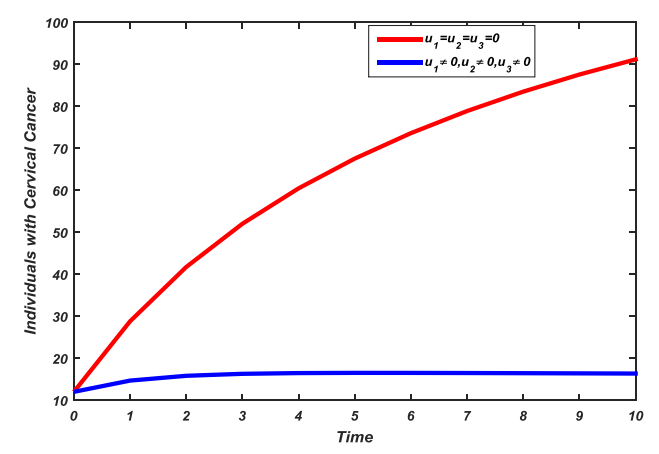

Fig. 8(c): Simulations of Cervical Cancer individuals with all controls

\section{Discussions and Conclusions}

In this study, a mathematical model formulated in [18]is modified by adding optimal control strategy. a. The wellpossedness of the modified model are performed. The study also obtained the basic reproduction number that governs the disease transmission from the largest eigenvalue of the nextgeneration matrix. The equilibria points of the model are obtained and their local as well as global stability condition is established. The model exhibits a backward bifurcation and the sensitivity analysis is performed. The optimal control problem is designed by applying Pontryagin maximum principle with three control strategies, namely, prevention strategy, treatment strategy and 
screening strategy. Numerical results for the human papillomavirus outbreak dynamics and its optimal control revealed that a combination of prevention, screening and treatment are the most effective strategy to eradicate the disease from the community.

Although eradication of HPV infection remain a challenge especially in developing countries, but from results of this study we recommend that, the government should introduce education programmers on the importance of voluntary and routinely screening on HPV infection. Also, there is need to increase the number of hospitals to deal with HPV infection as well as cancers to ensure that, many people have access to the facilities, because HPV infection in long run results into different types of human cancers which pose serious health problem. Moreover, the future work should consider; incorporating asymptomatic and treatment against HPV transmission dynamics in the model.

\section{References:}

[1] R.V. Barnabas, P. Laukkanen, P. Koskela, O. Kontula, M. Lehtinen, G.P. Garnett, "Epidemiology of HPV 16 and cervical cancer in Finland and the potential impact of vaccination: mathematical modelling analyses", PLoS Med. Vol.3, No. 5, 2006.

[2] A.S. Bergot, A. Kassianos, I.A. Frazer, D. Mittal, "New Approaches to immunotherapy for HPV associated cancers", OPEN ACESS, Cancers 2011, 346-3495; doi 10.3390/cancers 3033461 .

[3] D.R. Lowy and J.T. Schiller, "Prophylactic human papillomavirus vaccines", $J$ C Invest), Vol.116, No.5, 2006, pp.1167-1173.

[4] WHO list of priority medical devices for cancer management? Geneva: World Health Organization; 2017. Licence: CC BY-NC-SA 3.0 IGO.

[5] Ntekim A. "Cervical Cancer in Sub Sahara Africa, Topics on Cervical Cancer with an Advocacy for Prevention". Intech, 2012. Google Scholar.

[6] Jemal A, et al. "Global cancer statistics". $C A$ Cancer J Clin. Vol. 61, No. 2, 2011, pp. 6990. PubMed Google Scholar.

[7] Addis Ababa Cancer Registry Data (20122014).

[8] S.L. Lee and A.M. Tameru, "A Mathematical model of Human papillomavirus in the United States and its impact on cervical cancer", Ivyspring International Publisher J cancer.
Vol.

10.7150/jca.4161.

3,2012,pp.262-266 doi:

[9] L A Reynolds and E M Tansey, "History of Cervical Cancer and the role of the Human Papillomavirus, 1960-2000", London, on 13

[10] Ruanne V Barnabas, Päivi Laukkanen, Pentti Koskela, Osmo Kontula, Matti Lehtinen, and Geoff P Garnett. Epidemiology of HPV 16 and cervical cancer in finland and the potential impact of vaccination: mathematical modelling analyses. PLoS Med. Vol. 3, No. 5, 2006.

[11] Elamin H Elbasha, Erik J Dasbach, and Ralph P Insinga. Model for assessing human papillomavirus vaccination strategies. Emerg Infect Dis. Vol. 13, No.1, 2007, pp. 28-41.

[12] James P Hughes, Geoff P Garnett, and Laura Koutsky. The theoretical populationlevel impact of a prophylactic human papilloma virus vaccine. Epidemiology. Vol. 13, No.6, 2002, pp. 631-639.

[13] L. Ribassin-Majed and R. Lounes, A SIS Model for Human papillomavirus transmission. hal-00555733, 201, pp.1-14

[14] Al V Taira, Christopher P Neukermans, and Gillian D Sanders. Evaluating human papillomavirus vaccination programs. Emerg Infect Dis. Vol. 10. No.11, 2004, pp. 19151923.

[15] L. Ribassin-Majed, R. Lounes, S. Clemencos, Efficacy of vaccination against HPV infections to prevent cervical cancer in France: Present assessment and Pathways to improve vaccination policie. PLOS ONE. Vol.7, No.3, 2012, doi:10.-1371/journal, Pone 0032251

[16] Akram Ashyani, Hajimohammad Mohammadinejad, Omid RabieiMotlagh, "Stability Analysis of Mathematical Model of Virus Therapy for Cancer", Vol. 11, No. 2 ,2016, pp 97-110.

[17] Eshetu Dadi Gurmu and Purnachandra Rao Koya, Impact of Chemotherapy treatment of SITR Compartmentalization and Modelling of Human Papilloma Virus (HPV). IOSR Journal of Mathematics (IOSR - JM). Vol. 15, No.3, 2019, pp $17-29$.

[18] Eshetu Dadi Gurmu, Purnachandra Rao Koya. Sensitivity Analysis and Modeling the Impact of Screening on the Transmission Dynamics of Human Papilloma Virus (HPV). American Journal of Applied Mathematics. Vol. 7, No.3, 2019, pp. 70-79.

[19] C. Castillo-Chavez, B. Song. Dynamical models of tuberculosis and their applications. 
Math. Biosci. Eng. Vol.1, No. 2, 2004, pp.361-404.

[20] Chitnis, N., Hyman, J.M., and Cusching, J. M. Determining important Parameters in the spread of malaria through the sensitivity analysis of a mathematical Model. Bulletin of Mathematical Biology. Vol. 70 No. 5, 2008, 1272-12.

[21] W. H. Fleming and R. W. Rishel, Deterministic and Stochastic Optimal Control, Springer, New York, 1975.

[22] L. S. Pontryagin, V. G. Boltyanskii, R. V. Gamkrelidze, and E. F. Mishchenko, The Mathematical Theory of Optimal Processes, Wiley, New York, 1962.

[23] Suzanne Lenhart, John T.Workma. Optimal Control Applied to Biological Models (2007).

Contribution of Individual Authors to the Creation of a Scientific Article (Ghostwriting Policy)

Eshetu Dadi Gurmu has formulation model assumption, analysis and carried out the simulation. Dr. Boka Kumsa Bole has organized the introduction parts.

Prof. Purnachandra Rao Koya Maria has organized and executed the results of the formulated models.

\section{Creative Commons Attribution License} 4.0(Attribution 4.0 International, CC BY 4.0)

This article is published under the terms of the Creative Commons Attribution License 4.0 https://creativecommons.org/licenses/by/4.0/deed.en US 